\title{
Farside explorer: unique science from a mission to the farside of the moon
}

\author{
David Mimoun • Mark A. Wieczorek • Leon Alkalai • W. Bruce Banerdt • \\ David Baratoux • Jean-Louis Bougeret • Sylvain Bouley • Baptiste Cecconi • \\ Heino Falcke • Joachim Flohrer • Raphael F. Garcia • Robert Grimm • \\ Matthias Grott • Leonid Gurvits • Ralf Jaumann · Catherine L. Johnson • \\ Martin Knapmeyer • Naoki Kobayashi • Alexander Konovalenko • \\ David Lawrence · Mathieu Le Feuvre • Philippe Lognonné • Clive Neal • \\ Jürgen Oberst • Nils Olsen • Huub Röttgering • Tilman Spohn • \\ Susanne Vennerstrom • Graham Woan · Philippe Zarka
}

Received: 6 February 2011 / Accepted: 4 August 2011 / Published online: 27 October 2011 (C) The Author(s) 2011. This article is published with open access at Springerlink.com

Team members: full list available at http://farside.spacecampus-paris.eu/.

Mark Wieczorek was the Mission proposer.

D. Mimoun $(\bowtie)$

Université de Toulouse, ISAE 10 avenue Edouard Belin, BP 54032-31055, Toulouse Cedex 4, France e-mail: david.mimoun@isae.fr

M. A. Wieczorek · P. Lognonné

Institut de Physique du Globe de Paris, Univ Paris Diderot, Paris, France

\section{Alkalai · W. B. Banerdt}

Jet Propulsion Laboratory, California Institute of Technology, Pasadena, CA, USA

D. Baratoux · R. F. Garcia

Université de Toulouse, UPS-OMP, IRAP, Toulouse, France

D. Baratoux · R. F. Garcia

CNRS, IRAP, 14 avenue Edouard Belin, 31400 Toulouse, France

J.-L. Bougeret · B. Cecconi

Observatoire de Paris-CNRS, Paris, France

\section{S. Bouley}

Institut de Mécanique Céleste et de Calcul des Ephémerides,

Observatoire de Paris, Paris, France

\section{H. Falcke}

Radboud Universiteit Nijmegen, ASTRON, Nijmegen, The Netherlands

H. Falcke

MPIfR, Bonn, Germany 
Abstract Farside Explorer is a proposed Cosmic Vision medium-size mission to the farside of the Moon consisting of two landers and an instrumented relay satellite. The farside of the Moon is a unique scientific platform in that it is shielded from terrestrial radio-frequency interference, it recorded the primary differentiation and evolution of the Moon, it can be continuously monitored from the Earth-Moon L2 Lagrange point, and there is a complete lack of reflected solar illumination from the Earth. Farside Explorer will exploit these properties and make the first radio-astronomy measurements from the most radio-quiet region of near-Earth space, determine the internal structure and thermal evolution of the Moon, from crust to core, and quantify impact hazards in near-Earth space by the measurement of flashes generated by impact events. The Farside Explorer flight system includes two identical solarpowered landers and a science/telecommunications relay satellite to be placed in a halo orbit about the Earth-Moon L2 Lagrange point. One lander would explore the largest and oldest recognized impact basin in the Solar System-

J. Flohrer · M. Grott · R. Jaumann · M. Knapmeyer · J. Oberst · T. Spohn German Aerospace Center DLR, Berlin, Germany

R. Grimm

Southwest Research Institute, Boulder, CO, USA

L. Gurvits

Joint Institute for VLBI in Europe, Dwingeloo, The Netherlands

L. Gurvits

Department of Astrodynamics and Space Missions,

Delft University of Technology, Delft, The Netherlands

C. L. Johnson

Department of Earth and Ocean Sciences,

University of British Columbia, Vancouver, Canada

C. L. Johnson

Planetary Science Institute, Tucson, AZ, USA

N. Kobayashi

ISAS/JAXA, Sagamihara, Japan

A. Konovalenko

Institute of Radio Astronomy, Kharkov, Ukraine

D. Lawrence

Johns Hopkins University Applied Physics Laboratory, Laurel, MD, USA

M. Le Feuvre

Laboratoire de Planétologie et Géodynamique, Université de Nantes, Nantes, France

C. Neal

University of Notre Dame, Notre Dame, IN, USA 
the South Pole-Aitken basin-and the other would investigate the primordial highlands crust. Radio astronomy, geophysical, and geochemical instruments would be deployed on the surface, and the relay satellite would continuously monitor the surface for impact events.

Keywords Moon • Farside • Radio astronomy • Geophysics • Impact flux • ESA's Cosmic Vision program

\section{Introduction}

The farside hemisphere of the Moon (Fig. 1) is a unique place in the Solar System for a large range of scientific investigations. Being shielded from terrestrial radio-frequency interference, the farside of the Moon is the most radio-quiet environment in near-Earth space. The farside hemisphere faithfully records the primary differentiation of the Moon and hosts the largest recognized impact basin in the Solar System. From the Earth-Moon L2 Lagrange point, the farside hemisphere of the Moon is ideal for the continuous monitoring of meteoroid impacts with the lunar surface.

Farside Explorer aims to place two robotic landers on the farside hemisphere of the Moon and to put an instrumented relay satellite into a halo orbit about the Earth-Moon L2 Lagrange point. During the course of its 4year nominal mission, Farside Explorer would conduct three broad scientific investigations.

First, from the vantage point of the lunar surface, Farside Explorer would make the first extensive radio astronomy measurements in the most radioquiet region of near-Earth space. It would perform the first sky mapping at low frequencies and make pathfinder measurements of the red-shifted neutral hydrogen line that originates from before the formation of the first stars. Low-frequency radio bursts from our Sun would be quantified, as would

\footnotetext{
N. Olsen $\cdot$ S. Vennerstrom

Technical University of Denmark, Copenhagen, Denmark

H. Röttgering

Leiden Observatory, Leiden University, P.O. Box 9513,

Leiden, 2300 RA, The Netherlands

G. Woan

School of Physics and Astronomy, University of Glasgow, Glasgow, UK

P. Zarka

LESIA, Observatoire de Paris, CNRS, UPMC,

Université Paris-Diderot, Paris, France
} 
Fig. 1 Photograph of the eastern hemisphere of the Moon taken by the crew of Apollo 16. The ancient cratered farside hemisphere occupies about $3 / 4$ of the rightmost portion of this image, and a few of the nearside basaltic mare are seen to the far left

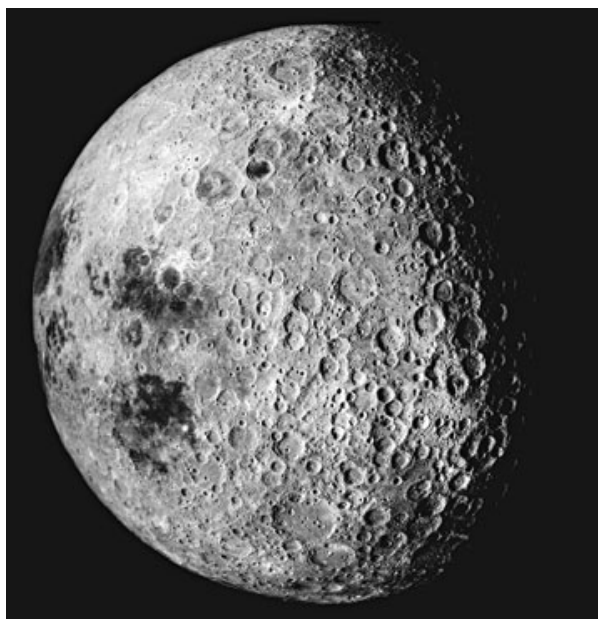

auroral emissions from the giant planets in the outer Solar System, pulsars, and the interaction of ultra-high energy cosmic rays with the lunar surface. The Farside Explorer radio astronomy experiment would be a pathfinder technology demonstration for a future radio array on the farside of the Moon.

Second, from the same landers, Farside Explorer would make precise geophysical measurements of the Moon's interior and measure the composition of its surface. From seismological, heat flow, and electromagnetic sounding measurements, these data would determine the bulk composition of the Moon, the thickness of its crust, the size and composition of its core, and the temperature profile of its interior. The surface geochemical data would provide critical ground truth measurements for the interpretation of orbital remotesensing data sets, and would help decipher the origin of two of the Moon's most prominent geologic provinces: the giant South Pole-Aitken basin and the primordial farside highlands.

Third, from the vantage point of the relay satellite, Farside Explorer would quantify near-Earth impact hazards by continuously monitoring the farside of the Moon for meteoroid impacts. Unspoiled by Earthshine and an intervening atmosphere, by the detection of impact flashes, this experiment would measure the Earth-Moon impact flux, the size-frequency distribution of impactors in near-Earth space, and spatial and temporal variations in the lunar impact rate during the lunar night. The measured impact times and locations would be used as known seismic sources for the seismology experiment, allowing for interior modeling from just two seismic stations.

Farside Explorer is an innovative mission that involves the development of soft-landing capabilities on airless bodies and that benefits from existing state-of-the-art geophysical and astronomical instrumentation in Europe. The scientific objectives of Farside Explorer are supported jointly by the radio astronomy and lunar science communities, address directly all four of the toplevel themes of ESA's Cosmic Vision program [5] (Table 1), and are identified 
Table 1 Farside Explorer relevance to Cosmic Vision science objectives

\begin{tabular}{|c|c|c|c|}
\hline Theme & Farside Explorer Relevance to CV theme & Measurement Objective & Instrument \\
\hline \multirow{4}{*}{$\begin{array}{l}\text { Theme } 1 \\
\text { What are the } \\
\text { conditions for planet } \\
\text { formation and the } \\
\text { emergence of life? }\end{array}$} & \multicolumn{2}{|c|}{1.1 From gas and dust to stars and planets } & 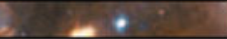 \\
\hline & $\begin{array}{l}\text { Farside Explorer will investigate the late stages of planetary } \\
\text { formation, including the event that formed the Earth-Moon system } \\
\text { and the impact that formed the South Pole-Aitken basin. }\end{array}$ & $\begin{array}{l}\text { Bulk composition of the Moon, crustal } \\
\text { thickness, core size, internal structure, } \\
\text { surface geology }\end{array}$ & $\begin{array}{l}\text { Seismometer, heat flow probe, } \\
\text { electromagnetic sounder, orbiting } \\
\text { magnetometer, surface camera }\end{array}$ \\
\hline & \multicolumn{3}{|l|}{ 1.3 Life and habitability in the Solar System } \\
\hline & $\begin{array}{l}\text { Farside Explorer will quantify impact hazards in near-Earth space, } \\
\text { elucidate the consequences of giant impact events that could } \\
\text { have frustrated the development of life, and constrain the manner } \\
\text { by which single-plate planets lose their heat. }\end{array}$ & $\begin{array}{l}\text { Size-frequency distribution and flux of near- } \\
\text { Earth objects, crustal thickness within the } \\
\text { South Pole-Aitken basin, heat flow }\end{array}$ & $\begin{array}{l}\text { Impact flash camera, seismometer, } \\
\text { heat flow probe }\end{array}$ \\
\hline \multirow{8}{*}{$\begin{array}{l}\text { Theme } 2 \\
\text { How does the Solar } \\
\text { System work? }\end{array}$} & \multicolumn{3}{|c|}{ 2.1 From the Sun to the edge of the Solar System } \\
\hline & $\begin{array}{l}\text { Farside explorer will measure low-frequency radio emissions from } \\
\text { the Sun, uncontaminated by terrestrial radio-frequency } \\
\text { interference. }\end{array}$ & Low-frequency radio monitoring of the Sun & Radio astronomy receiver \\
\hline & $\begin{array}{l}\text { Farside Explorer will quantify how the solar wind interacts with } \\
\text { atmosphereless bodies. }\end{array}$ & $\begin{array}{l}\text { Electromagnetic measurements from the } \\
\text { surface and orbit }\end{array}$ & $\begin{array}{l}\text { Electromagnetic sounder, orbiting } \\
\text { magnetometer }\end{array}$ \\
\hline & $\begin{array}{l}\text { Farside Explorer will investigate how planets differentiate into a } \\
\text { crust, mantle and core, and how tectonic processes work on } \\
\text { single-plate planets. }\end{array}$ & $\begin{array}{l}\text { Internal structure of the Moon, surface heat } \\
\text { flow }\end{array}$ & $\begin{array}{l}\text { Seismometer, heat flow probe, } \\
\text { electromagnetic sounder, orbiting } \\
\text { magnetometer }\end{array}$ \\
\hline & \multicolumn{3}{|l|}{ 2.2 The giant planets and their environments } \\
\hline & $\begin{array}{l}\text { Farside Explorer will measure the magnetospheric emissions of } \\
\text { the giant planets, their time variations, and the coupling with their } \\
\text { satellites. }\end{array}$ & $\begin{array}{l}\text { Low-frequency radio monitoring of the outer } \\
\text { planets, uncontaminated by solar and } \\
\text { terrestrial emissions }\end{array}$ & Radio astronomy receiver \\
\hline & \multicolumn{3}{|l|}{ 2.3 Asteroids and other small bodies } \\
\hline & $\begin{array}{l}\text { Farside Explorer will constrain the flux, size-frequency } \\
\text { distribution, and physical properties of small near-Earth objects. }\end{array}$ & $\begin{array}{l}\text { Monitoring of visual and infrared impact } \\
\text { flashes }\end{array}$ & Impact flash camera, seismometer \\
\hline Theme 3 & \multicolumn{3}{|l|}{ 3.3 Matter under extreme conditions $\times$} \\
\hline $\begin{array}{l}\text { What are the } \\
\text { fundamental physical } \\
\text { laws of the Universe? }\end{array}$ & $\begin{array}{l}\text { Farside Explorer will detect interactions between ultra high energy } \\
\text { cosmic rays and the lunar surface. }\end{array}$ & Low-frequency radio measurements & Radio astronomy receiver \\
\hline \multirow{2}{*}{$\begin{array}{l}\text { Theme } 4 \\
\text { How did the Universe } \\
\text { originate and what is } \\
\text { it made of? }\end{array}$} & \multicolumn{3}{|c|}{ 4.1 The early Universe } \\
\hline & $\begin{array}{l}\text { Farside Explorer will investigate the cosmological dark ages } \\
\text { through the red-shifted neutral hydrogen } 21-\mathrm{cm} \text { line. }\end{array}$ & $\begin{array}{l}\text { Low-frequency radio measurements } \\
\text { uncontaminated by solar and terrestrial } \\
\text { emissions }\end{array}$ & Radio astronomy receiver \\
\hline
\end{tabular}

as top priorities in the United States planetary science decadal survey [13]. Farside Explorer would participate in the internationally renewed exploration of Earth's nearest celestial neighbor.

\section{Science objectives}

The science objectives of Farside Explorer are conceived to exploit the unique environment offered by the farside hemisphere of the Moon. Three primary investigations are dictated by the properties of this platform.

The farside of the Moon is a unique environment for low-frequency radio measurements On Earth, several sources of radio-frequency interference exist, such as radio broadcasts and lightning. Furthermore, extra-terrestrial signals with frequencies below $10-20 \mathrm{MHz}$ cannot be studied since they are reflected off of, or are severely distorted by, the Earth's ionosphere. The farside of the Moon does not suffer from either of these problems given that the Earth and its orbiting communications satellites are not in direct view from this platform; it is the ideal place to investigate one of the last unexplored regions of the electromagnetic spectrum (Fig. 2) [27]. 

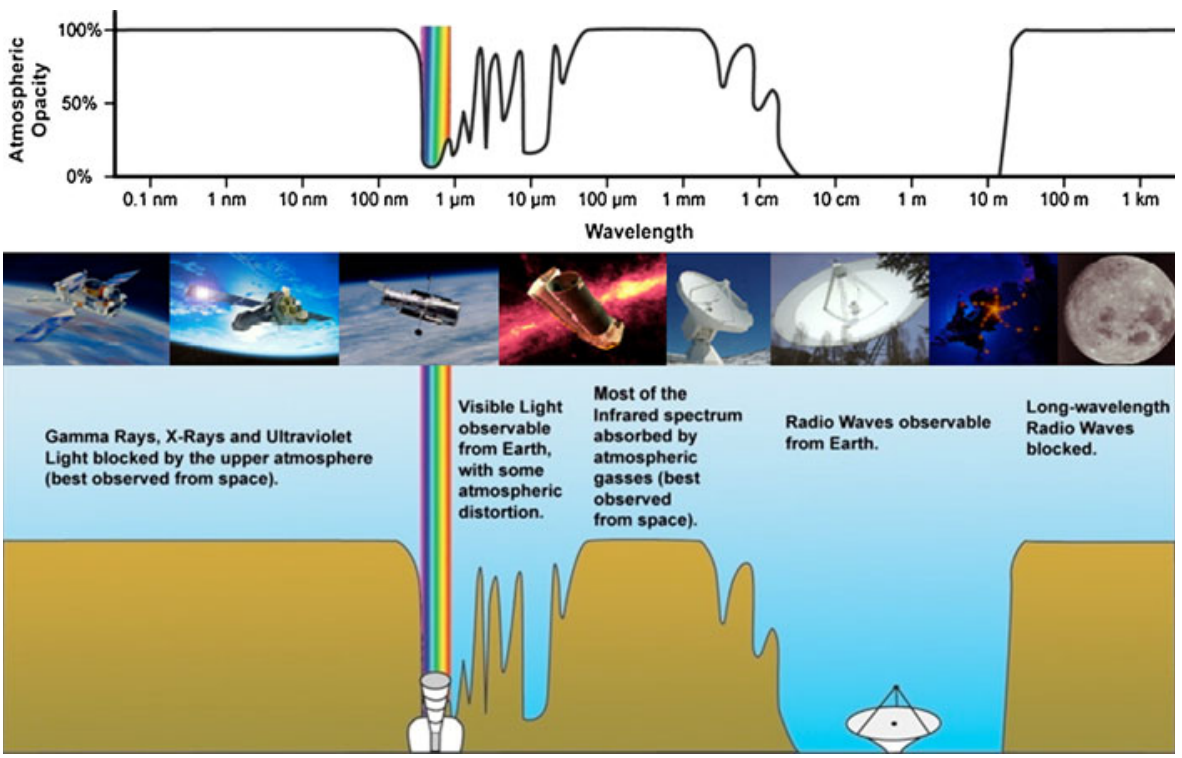

Fig. 2 Atmospheric and ionospheric effects allow only a small portion of the electromagnetic spectrum to be investigated from Earth

From the vantage point of the lunar farside, a single (dual-polarized) antenna would make extraordinary measurements: Low-frequency radio bursts from the outer giant planets of our Solar System would be monitored, the redshifted neutral hydrogen (HI) line that originates from before the formation of the first stars would be investigated, and the interaction of the Moon's surface with ultra-high energy cosmic rays that exceed the energies of modern particle accelerators would be detected. Given the broad interest in the creation of a low-frequency aperture synthesis radio array on the Moon's surface, such pioneering low-frequency interferometric radio measurements would demonstrate the feasibility of such a large-scale project.

The farside of the Moon is a unique laboratory for investigating planetary formation and evolution The Earth-Moon system is believed to have formed by a giant impact between the nascent Earth and a Mars-sized object. This event led to the formation of globe-encircling magma oceans on both bodies and contributed to their primary differentiation and core formation. In contrast to the Earth, the interior structure and composition of the Moon have evolved little since this time. Both the Earth and Moon suffered subsequently the consequences of large impact events, but this important period of time has been almost totally erased from the Earth's surface, whereas it is ideally preserved on the Moon. The Moon is an end-member of terrestrial planetary evolution and is the nearest celestial object to have preserved the record of early planetary processes. 
Though the geophysical investigations of the Apollo era provided tantalizing clues to lunar formation, differentiation, and evolution, in retrospect, the Apollo nearside landing sites were not ideal for this purpose. It is now recognized that these measurements are biased by their proximity to an atypical geological province called the Procellarum KREEP Terrane (Fig. 3) [28]. The most prominent terrane that records the formation of the primordial crust is located largely on the farside hemisphere, as is the largest and oldest recognized impact basin in our Solar System, the South Pole-Aitken basin. The farside hemisphere of the Moon is the best place to investigate the relics of planetary differentiation that are recorded beneath its surface.

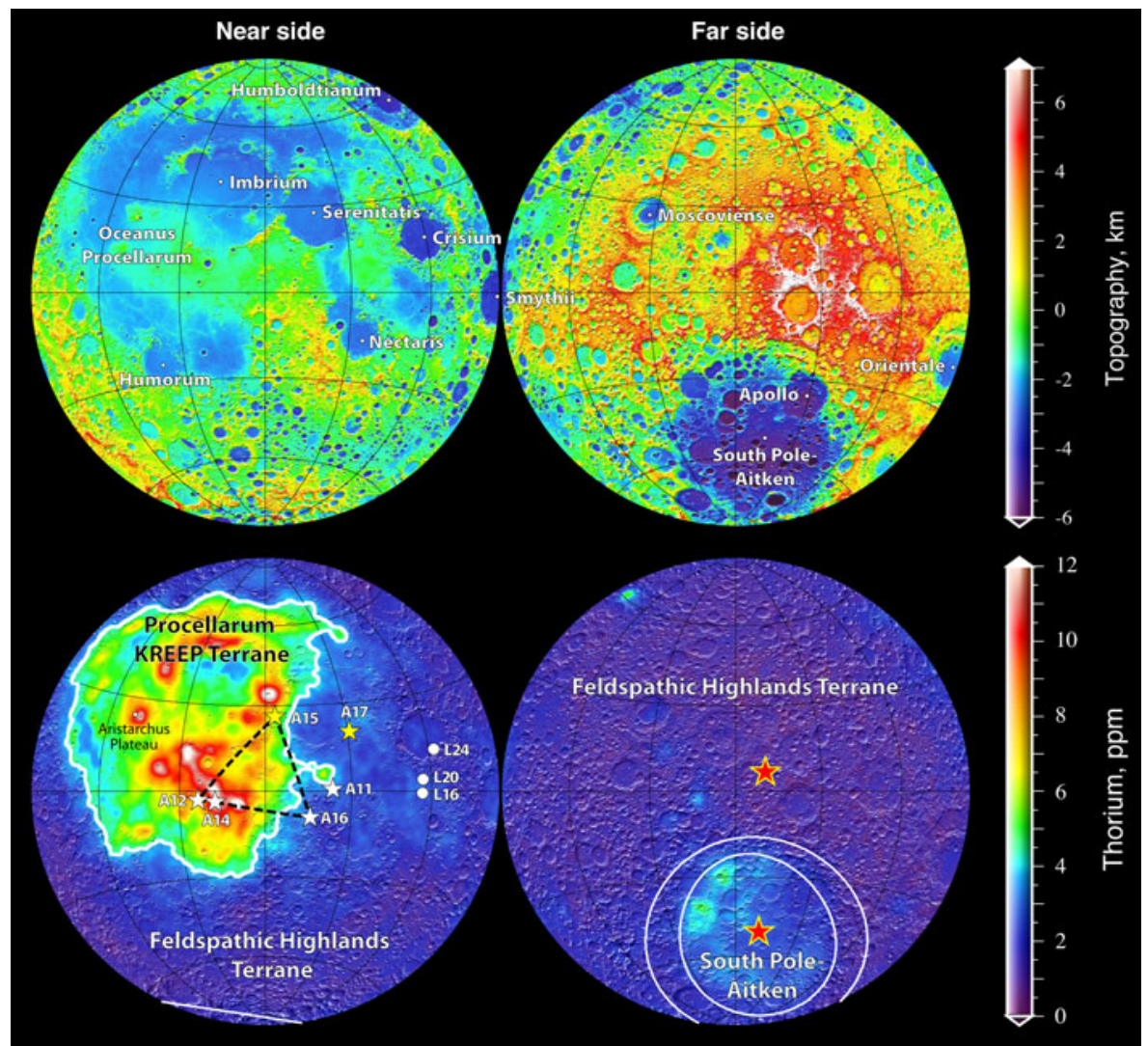

Fig. 3 Topography of the Moon [62] and surface abundance of thorium [34]. The thick white contour at $4 \mathrm{ppm}$ thorium delineates the approximate confines of the Procellarum KREEP Terrane [28], and the thin white ellipses on the farside outline the floor and structural rim of the South Pole-Aitken basin [18]. Stars and circles on the nearside represent the Apollo and Luna sample return stations, respectively. Yellow stars correspond to the locations where two heat-flow measurements were made, and the black dashed lines connect the four Apollo stations containing seismometers that operated in a network fashion. The two red stars on the farside hemisphere mark the proposed landing sites for Farside Explorer. Images of the near- and farside-hemispheres are displayed using Lambert azimuthal equal area projections 
Impacts between near-Earth objects and the Moon are ideally monitored from the Earth-Moon L2 Lagrange point From the vantage of the L2 Lagrange point, about $60,000 \mathrm{~km}$ above the lunar surface, a camera could make measurements of the visible and thermal flashes generated by meteoroid impacts that would be superior to those made on the nearside hemisphere from Earth [e.g., 51, 66]. These observations would not be affected by local weather conditions, and given the lack of an intervening atmosphere, critical nearinfrared observations would be possible. Since there is no Earthshine light pollution on the farside of the Moon, and since L2 is significantly closer to the Moon than is the Earth, impact flashes would be detectable for objects considerably smaller than could be seen from a comparable optical system on the Earth's surface.

In combination with simultaneous seismic measurements on the surface, these impact flash measurements will make it possible to constrain both the impact flux and size-frequency distribution of centimeter to meter sized near-Earth objects. Through long-term monitoring, both temporal and spatial variations in the impact flux on the Moon would be quantified [35], allowing for a better understanding of the origin of these objects.

Farside Explorer would exploit these three unique properties of the Moon's farside hemisphere and would make synergistic measurements from orbit and the surface. Two identical landers would contain a suite of state-ofthe-art astronomical, geophysical, and geochemical instruments, including: a radio astronomy experiment, a long- and short-period seismometer for lunar internal structure and impact investigations, a heat flow probe for thermal evolution and bulk composition studies, an electromagnetic sounder for electrical conductivity and temperature profile estimation, a geochemical experiment to provide ground truth measurements for orbital remote sensing data sets, and surface cameras for landing site characterization and monitoring. The orbiter would contain an impact flash camera for the monitoring of impact hazards, and a magnetometer for electromagnetic sounding of the lunar interior.

The surface payload of Farside Explorer contains all instruments that the International Lunar Network [45] identified as core instruments for any future geophysical network. (Laser retroreflectors, which were identified as core instruments for nearside stations, are not applicable to farside stations.) The International Lunar Network also ranked radio astronomy and surface geochemistry as two of the most important non-core instruments for such a network. Farside Explorer will investigate two of the most prominent geological provinces of the Moon [28], the ancient Feldspathic Highlands Terrane that dates from the time of magma ocean crystallization, and the South PoleAitken basin, which is the largest and oldest unequivocal impact structure on any terrestrial planet (Fig. 3). These two targets are among the prime landing sites selected by the International Lunar Network [71].

The following sections describe the radio astronomy, lunar science, and impact monitoring objectives. 


\subsection{Radio astronomy}

Current knowledge The Moon, in contrast to Earth, has an extremely thin ionosphere that allows radiofrequency measurements down to $500 \mathrm{kHz}$ during the day, and even lower during the night [68]. Its farside hemisphere is shielded from terrestrial radio interference, satellite interference, lightning, and auroral emissions, and during the lunar night the farside is also shielded from solar radio emissions. For these reasons, the farside of the Moon has been considered to be an ideal site for low-frequency radio astronomy since the 1960s [4, 27]. The two Radio Astronomy Explorer satellites launched in 1968 and 1973 are the only spacecraft to have made low frequency radio measurements in the frequency range of 0.02 to $13.1 \mathrm{MHz}$ [2]. From the collected data (total flux only), these spacecraft could study only solar, Jovian and terrestrial radio emissions [30].

Radio astronomy science goals Astrophysics at frequencies below $10-20 \mathrm{MHz}$ is today almost entirely unexplored. Though solar and planetary studies can be performed from spacecraft above the Earth's ionosphere, these observations are made in permanent view of strong sources from the Sun, Jupiter, and Earth. No dedicated measurements exist from a place as quiet as the lunar farside. From this location, the only sensitivity limitation would come from the bright galactic radio background, and this could be lowered by broad frequency and long time integrations. Being an unexplored portion of the electromagnetic spectrum, low-frequency radio measurements from the farside of the Moon will elucidate many processes (Fig. 4).

Cosmology The frequency range less than $40 \mathrm{MHz}$ corresponds to the redshifted neutral hydrogen (HI) $21-\mathrm{cm}$ line at $\mathrm{z} \geq 35$, and thus to the so-called "dark ages" that preceded the epoch of reionization in the Universe. Theoretical modeling of the global HI signature predicts a specific spectral variation with amplitudes about $10^{-6}$ times the sky background [27, 59]. Single-dipole detection of this weak global signal would be difficult due to the variations with time, space and frequency of the foreground sources. Nevertheless, upper bounds would be provided, and the identification and study of the foreground signals at these frequencies is an important astronomical objective in itself.

Sky mapping Combining the time-variable occultation of one-half of the sky by the Moon, goniopolarimetric measurements by each lander, and inversion of series of interferometric measurements (complex visibilities obtained by correlation of time-shifted signals from the 2 landers), radio sky maps will be built at several frequencies together with an accurate spectrum of the sky background intensity and polarization [15]. This mapping, with an expected resolution of degrees to tens of degrees, would provide information on distant radio galaxies and large-scale structures such as clusters with radio halos and cosmological filaments.

Solar physics The frequency range of $0.1-40 \mathrm{MHz}$ corresponds to plasma frequencies in the solar corona between about two and several tens of solar 


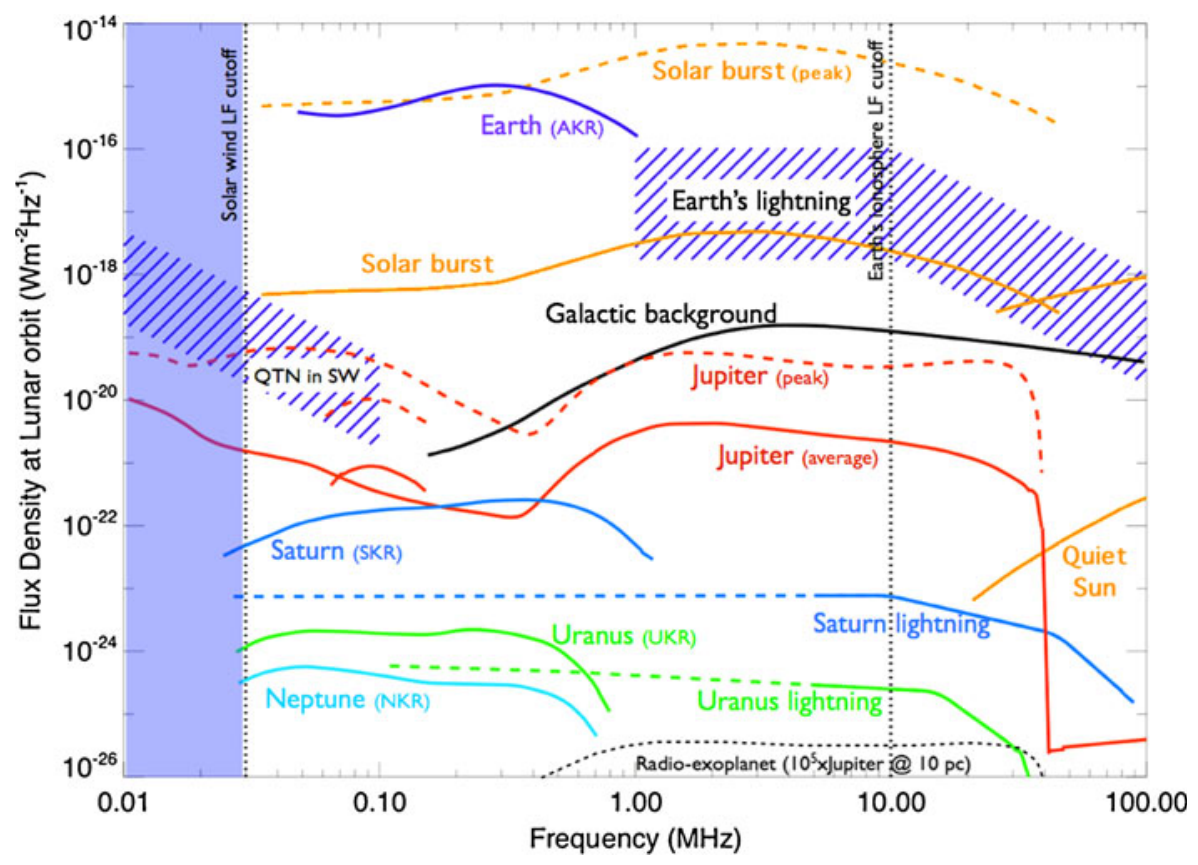

Fig. 4 Planetary, solar and predicted exoplanetary radio emission levels on the surface of the Moon, compared to the galactic background detected with a single dipole. From the farside, no terrestrial signal (dark blue) pollutes the observations above $\sim 0.5 \mathrm{MHz}$. During the lunar night, no solar emissions (orange) are present, and observations can be extended down to the solar wind cutoff of $\sim 30 \mathrm{kHz}[80]$

radii from the center of the Sun [42]. As solar type II and III radio bursts are emitted at 1-2 times the plasma frequency (such as on shock fronts at stream interfaces in the solar wind, coronal mass ejections, and by energetic electron beams), occurrence and evolution of these structures would be monitored throughout the solar corona. Goniopolarimetry would provide directional information on the emitting structures [12], and Farside Explorer observations would be used synergistically with spacecraft observations at similar frequencies and ground-based observations at higher frequencies [8].

(Exo-)planetary magnetospheres With two crossed dipoles, detection of magnetospheric radio emissions from all giant planets [78] would be possible on a regular basis. Long-term observations would permit fundamental studies such as the accurate determination of planetary rotation periods and their variations [77]. They would also address, by the modulations of radio emissions by natural satellites and solar wind strength, magnetospheric dynamics and electrodynamic coupling with satellites. Farside Explorer radio observations would provide the first measurements of Uranus and Neptune since Voyager 2. For Jupiter, these measurements would allow investigation of the plasma torus of Io, and possibly to monitor Io's volcanism and detect secular magnetic field variations. For Saturn, Farside Explorer would extend the long series 
of Cassini measurements, permitting seasonal or secular studies. Though exoplanetary radio emissions would not be detectable with a few dipoles, Farside Explorer measurements would quantify the sensitivity reachable as a function of frequency, and set the context for future exoplanet low-frequency radio searches [79].

Pulsars and radio propagation Due to their periodic nature, radio emissions from a few known intense pulsars should be detectable by an adequate processing of waveform measurements following long integrations $[10,56]$. These challenging observations would provide us with the first measurements of a few pulsar spectra down to very low frequencies, and allow us to quantify propagation effects (such as dispersion and temporal broadening) down to increasingly lower frequencies. Temporal broadening of pulses, if found to depart from Kolmogorov-induced $\lambda^{4.4}$ variations, could reveal a cutoff in the distribution of turbulence in the interstellar medium, and open the possibility of low-frequency radio observations of variable sources at lower frequencies than expected.

Transient events Radio transient events that may be produced by the interaction of ultra high energy cosmic rays (UHECRs) with the Moon's surface would be investigated by the radio astronomy experiment. These events should produce a cascade of particles propagating in the regolith, leading to Cerenkov emission. Particle shower lengths of a few meters imply a coherent radio pulse of 10-1000 nanoseconds and a spectral increase between 1-100 MHz. Farside Explorer measurements should allow to detect UHECRs within $5 \mathrm{~km}$ of each lander at a rate of $\sim 1$ event/year above $10^{20} \mathrm{eV}$.

High-energy neutrinos behave like UHECRs, but with a much deeper penetration depth and detector volume. Not being deviated by interstellar fields, their direction of arrival points towards their original source, and if detected by both landers, this would allow determination of a plane containing the direction of arrival. These studies could eventually be advantageously competitive with the largest existing cosmic rays detectors of neutrino experiments in Antarctica [27].

Local environment studies Farside Explorer would monitor the peak plasma frequency above the landers as a function of lunar local time, solar activity, and traversal of the Earth's magnetotail. Charging of the lunar regolith due to ultra-violet (UV) illumination, charged particles, or micrometeoritic impacts could result in detectable electrostatic discharges.

Pathfinder measurements for an eventual lunar array A longer-term goal in radio astronomy is the construction of a large low-frequency radio array on the Moon's farside hemisphere. Farside Explorer will provide invaluable pioneering measurements that will influence the design of such an array.

Radio astronomy measurement requirements A sensitive radio receiver on each lander, performing spectrometry, goniopolarimetry and waveform capture, connected to dipole antennas, would fulfill the requirements of all radio astronomy science goals. Electrical properties of the lunar regolith and uppermost crust, measured in situ and in the lab on returned samples, indicate 
that radio signals can penetrate to kilometer depths without strong scattering. The low conductivity and moderate dielectric constant should cause only a small distortion of the skyward beam pattern.

The radio-spectrometer mode would be used as a baseline for monitoring the lunar environment from $16 \mathrm{kHz}$ to approximately $40 \mathrm{MHz}$. Its goniopolarimetric mode would be the basis for separating solar, planetary and other emissions detectable with limited integration over sky background fluctuations.

Waveform capture, with an instantaneous bandwidth of several $\mathrm{MHz}$, would be the basis of interferometric measurements for constraining the extent of sources intense enough to be localized by goniopolarimetry. Synchronized waveform snapshots from the two landers would be sent to the relay satellite for correlation, and complex visibilities would be stored to perform imaging of the static radio sky. Individual waveform snapshots would enable studies requiring high time resolution (like burst detection) or high sensitivity (such as pulsar detection and study of propagation effects), for which they could be processed on the relay satellite, or onboard each lander in order to increase their duty cycle. Time coincidence between radio pulses detected at the two landers would also be used. Localization of the static lander positions, as required for the interferometric measurements, will be made possible (with an increasing precision level) by the use of Doppler radio communication between the relay satellite and landers, then by the use of existing sub-meter resolution orbital images of the landing site with images taken from the lander, and finally through the observation of known radio sources. The unique character of lunar farside radio measurements is their extreme sensitivity due to the extreme radio quietness of the site. Special care must thus be taken to achieve high electromagnetic cleanliness of the lander's payload and communication links, as has been done on missions such as Ulysses, Stereo, BepiColombo, and to measure the effective pollution brought by the mission itself to the pristine lunar environment.

\subsection{Lunar science}

The Moon is the only terrestrial object for which we have samples from known locations, geophysical data from dedicated stations on the surface, and observations from field geologists. From these data, the origin of the Moon from a giant Mars-sized impact with the early Earth, the existence of a globeencircling magma ocean that formed an ancient primary flotation crust, the existence of distinct geologic terranes, and a 3 billion year record of volcanic activity have been elucidated [29].

Measurements of the lunar interior were made from the Apollo Lunar Surface Experiment Package (ALSEP) that included a passive seismic experiment, heat flow probes, magnetometers, and laser retroreflectors. However, because of the limited extent of the ALSEP network, and the unfortunate placement of these stations near the boundary of the two most prominent terranes (see Fig. 3), the lunar interior remains relatively unknown (Fig. 5). Farside Explorer has two primary lunar science goals: (1) to determine the 


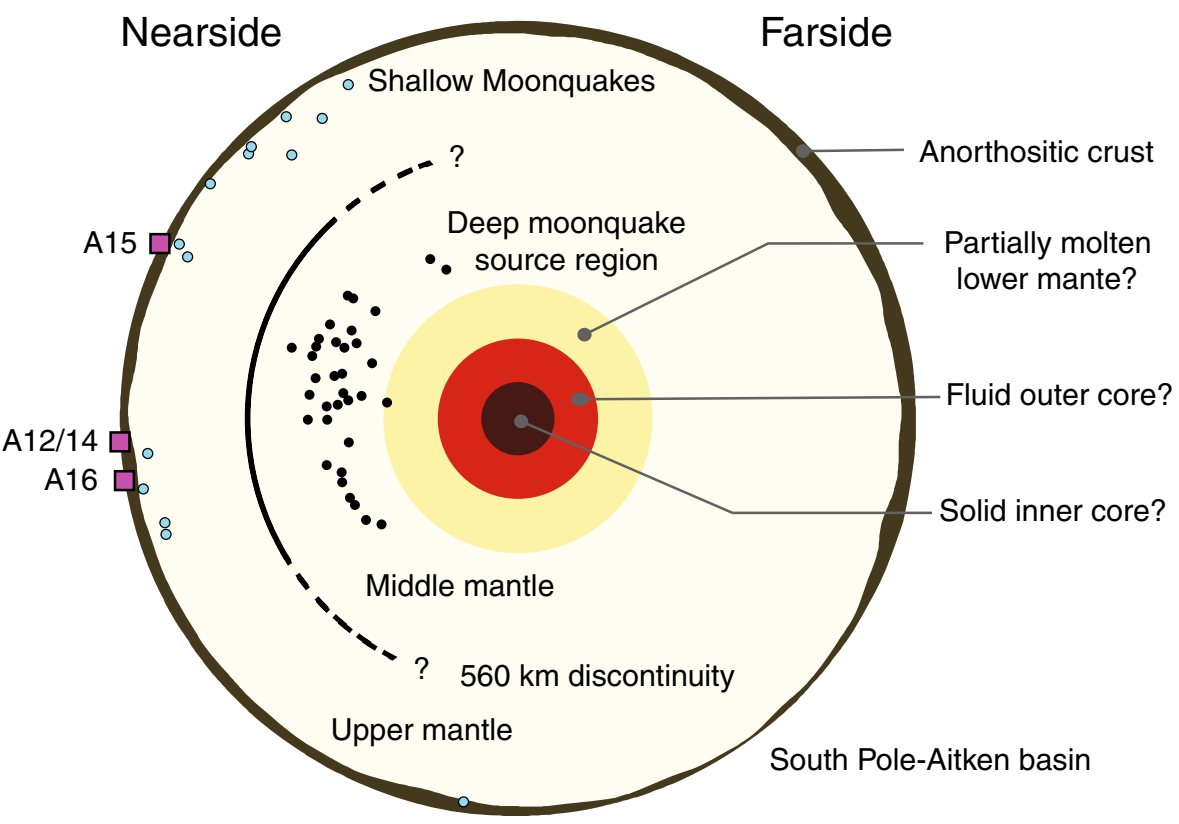

Fig. 5 Schematic diagram (to scale) of the Moon's interior structure. Shown are the Apollo seismic stations (squares), all shallow moonquakes (blue circles), the deep moonquake source regions that are periodically activated by Earth-raised tides (black circles), inferred variations in crustal thickness, and a possible discontinuity $\sim 500 \mathrm{~km}$ below the surface. The structure below the deep moonquake source region is constrained only by indirect means, and little is known about the farside hemisphere. Image from [73]

internal structure of the Moon, from crust to core, and (2) to elucidate its thermal evolution. A secondary goal is to determine the surface composition at two locations far from the Apollo and Luna sampling stations.

\subsubsection{Internal structure of the moon}

Current knowledge Much of our knowledge of the Moon's internal structure comes from analyses of the Apollo seismic data that were collected from 4 stations in network mode between 1971 and 1977 [46]. The Apollo instruments were extremely sensitive, but the limitations of late 1960s digital technology, the narrow frequency range, and the small footprint of the network near the sub-Earth point placed severe limitations on seismic data interpretation. Natural source electromagnetic sounding is another geophysical method that was used to sense the deep interior of the Moon. From the analysis of magnetic field data collected simultaneously on the surface and from orbit, it is possible to recover the Moon's electrical conductivity profile, which is sensitive to interior temperature and composition. The following paragraphs summarize what has been inferred about the Moon from the ALSEP data. 
Crust A range of crustal thickness estimates has been proposed beneath the Apollo seismic network, from 60, to 45, to $30 \mathrm{~km}$ [40]. This partially reflects improvements in computing capacity since the Apollo era, but it also reflects different interpretations allowed by limitations of the seismic data. Regardless of these uncertainties, the Apollo seismic network straddled the boundary of two distinct geologic provinces and the crustal properties of these two terranes were not observed unambiguously. It is possible to infer spatial variations in crustal thickness using gravitational and topographic data obtained from orbit, but these methods need to be anchored to ground-truth values obtained from seismic measurements.

Mantle A fundamental question in lunar science is how much of the Moon was molten immediately after its accretion. An apparent seismic discontinuity was reported initially about $500 \mathrm{~km}$ below the surface, and this has been interpreted by some to mark the base of the lunar magma ocean [73]. More recent models without discontinuities are also compatible with the data [40], but even if this discontinuity does exist, the limited footprint of the Apollo seismic network leaves open the question as to whether it is a widespread feature of the mantle.

Core Many studies over the past 30 years have attempted to constrain the nature of the Moon's core using its moment of inertia, induced magnetic signature, rotational dissipation observed in the lunar laser ranging data, and the depletion of highly siderophile elements in the mare basalt source region [73]. These studies generally conclude that the Moon possesses a liquid metallic core (Fe with some $\mathrm{Ni}, \mathrm{S}$, and $\mathrm{C}$ ) that is less than $400 \mathrm{~km}$ in radius. However, the inferences drawn from such studies are not unique. The core size inferred from the magnetic data depends upon the assumed electrical conductivity, and the core size inferred from lunar laser ranging data depends upon the assumed density, viscosity, and core-mantle boundary shape. Though the detection of reflected energy from the core in the Apollo seismic data was reported recently $[17,70]$, these analyses required the stacking and filtering of multiple events, and is also not free of interpretation.

Bulk composition Previously reported bulk silicate Moon compositions vary by almost 4 wt. $\%$ in $\mathrm{Al}_{2} \mathrm{O}_{3}$ and $\mathrm{FeO}, 10$ wt. $\%$ in $\mathrm{MgO}$, and 3 wt. $\%$ in $\mathrm{CaO}$ [69]. Better determination of the geophysical parameters of the lunar core, mantle, and crust would provide improved bulk compositional constraints that could be used to test hypotheses regarding the Moon's origin.

Remaining questions Several questions remain concerning the seismicity and internal structure of the Moon:

- Do deep moonquakes occur on the farside hemisphere? Or is the farside seismically inactive?

- What is the composition of the deep mantle?

- What is the size and composition of the Moon's core? And does the Moon have a solid inner core? 
- Do seismic discontinuities exist in the mantle? And how are these related to crystallization of the lunar magma ocean?

- How thick is the lunar crust? And did the South Pole-Aitken impact event excavate into the mantle?

These questions would be resolved by data obtained from the seismic and electromagnetic sounding experiments on Farside Explorer.

Seismology science objectives Without a priori information, a minimum of three seismic stations is required to estimate the location and time of a seismic source, and the average $\mathrm{P}$ - and $\mathrm{S}$-wave velocities between the source and stations. A novel aspect of the Farside Explorer seismic experiment is the use of seismic source locations and times from impact flashes observed by the dedicated impact monitoring experiment and the a priori knowledge of deep moonquake source locations determined from the ALSEP experiment. With the location of the seismic event known, it is possible to constrain the interior structure of the Moon with even a single seismic station.

Lunar impactors as small as $0.1 \mathrm{~kg}$ are marked by light flashes detectable by Earth-based telescopes on the nearside hemisphere $[51,66]$ and by the Farside Explorer dedicated impact flash camera on the farside. Simulations show that the seismology experiment would detect about 400 of these impact events per year, and this active seismic experiment would allow very precise tomographic studies of the crust and upper mantle.

The internal seismic activity of the Moon during the Farside Explorer mission can be constrained with confidence using the known 6-year periodicity of the deep moonquake source regions. 2800 events are expected that would exceed the Apollo detection threshold during a 4-year mission, and for a conservative detection level 5 times more sensitive than Apollo, each year about 10,000 deep moonquakes are expected. The source locations of many of the deep moonquake nests on the lunar near side have already been determined from the Apollo data, and it is known that these are repeatedly activated with tidal periodicities. Using the prior information of these source locations, it is possible to invert for internal structure using only two seismic stations.

A key science objective is to determine the size of the Moon's core, and the Farside Explorer strategy is based on the refraction and reflection of seismic waves by the core from the known deep moonquake nests (Fig. 6). This is accomplished by observations of the PKP phase, a P-wave that travels through the core, and the $\mathrm{ScS}$ phase, an S-wave reflected at the core-mantle boundary. These phases were not directly detected by the Apollo era instruments, but are above the detection threshold of modern planetary seismometers (Fig. 7). The locations of the deep moonquake nests on the near side are well known, and the ideal configuration of the Farside Explorer network should allow for the detection of a solid inner core, if one is present.

The Farside Explorer seismology instrument is superior to the Apollo seismometers [44]. It has a much wider bandwidth, and contains 3 long period 


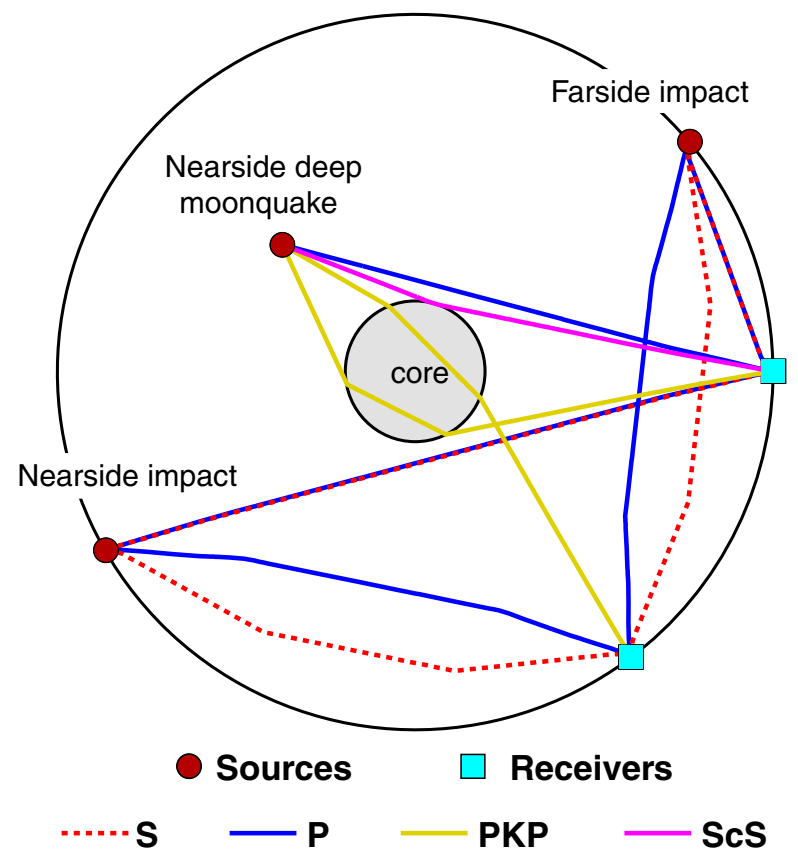

Fig. 6 Example $\mathrm{P}$ and $\mathrm{S}$ seismic ray paths associated with meteoroid impacts and deep moonquakes (using the velocity model of [46]). Meteoroid impact locations and times will be provided by the observation of impact flashes, allowing interior modeling with even a single seismic station. The locations of many of the nearside deep moonquake source regions are known from analyses of the Apollo seismic data, and this allows for interior modeling from just two seismic stations. Seismic waves from the nearside deep moonquakes that are refracted (PKP) and reflected (ScS) from the core are ideally observed on the farside of the Moon. Core phases from the more frequent impact events will be too weak to be detected

and 3 short period sensors in comparison to the 4 sensors of Apollo. The very large dynamic range allows precise sampling of waveforms below the instrument noise floor, allowing the stacking of deep moonquake signals. It realizes a factor of $\sim 10-20$ increase in sensitivity in the body wave band $0.1-$ $10 \mathrm{~Hz}$ and explores for the first time the $10-40 \mathrm{~Hz}$ and $<0.1 \mathrm{~Hz}$ bands.

Electromagnetic sounding science objectives Electromagnetic sounding uses measurements of the magnetic and/or electric field over a range of frequencies to estimate impedance (e.g., transfer functions) that provide information on the electrical conductivity beneath the surface. From these measurements, the size of a high-conductivity core would be determined, depth variations in composition would be delineated, and the temperature of the mantle would be constrained using laboratory measurements of the temperature dependence of electrical conductivity [16, 23, 25, 31, 63].

A variety of approaches are possible to measure the frequency dependent electromagnetic impedance [20]. The Apollo-era studies used the distantly 


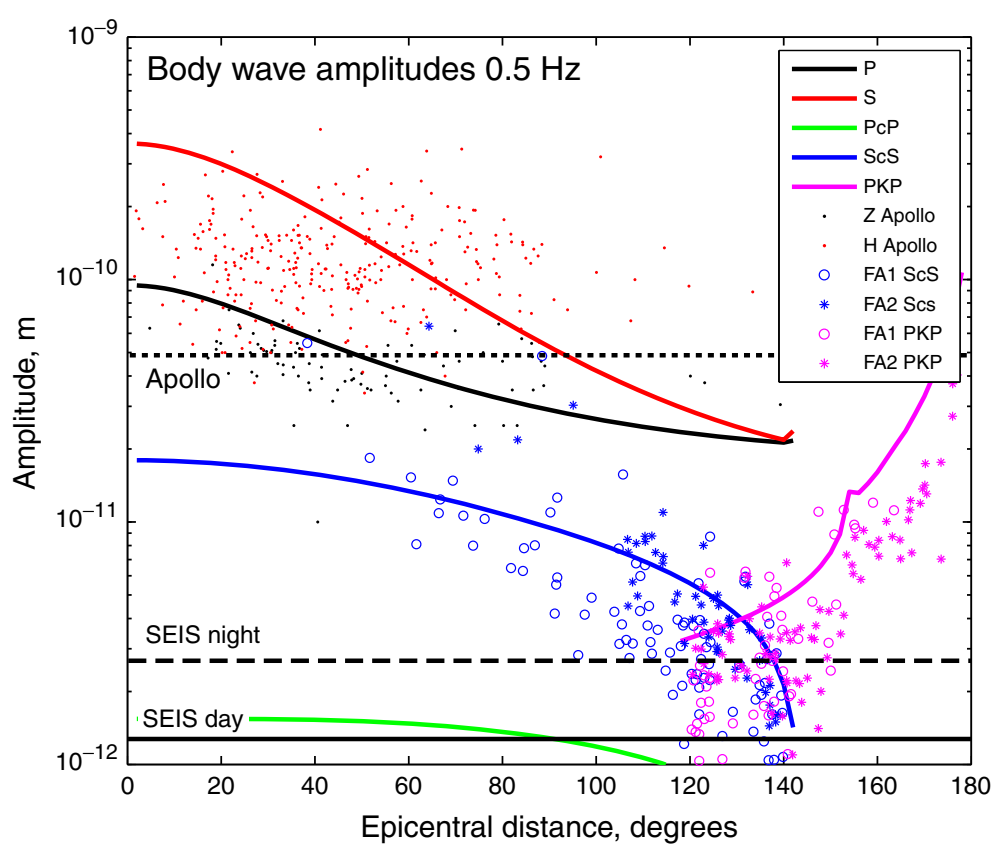

Fig. 7 Detection of seismic core phases by Farside Explorer. Red and black dots show the mean $\mathrm{P}$ and $\mathrm{S}$ amplitudes of the deep moonquakes detected by the Apollo stations as a function of epicentral distance; blue and magenta symbols show the expected amplitudes at the two Farside Explorer stations (FS1 and FS2) for reflected (ScS) and refracted (PKP) core arrivals from the same near-side deep moonquake nests. Solid colored lines represent average expected amplitudes. The horizontal dotted, dashed, and solid lines are the instrument noise levels for the Apollo and Farside Explorer seismometers (SEIS) during the day and night. A large fraction of the core arrivals will be detectable by Farside Explorer

orbiting Explorer 35 to determine the source magnetic field, and compared this to surface measurements that represent the sum of the source and induced fields [24]. This transfer function technique will be repeated for Farside Explorer using magnetometers on the two landers and relay satellite.

A second technique, geomagnetic depth sounding, reconstructs the impedance from the vertical magnetic field and the horizontal gradient of the horizontal field [19]. A spatial array of magnetometers is necessary to determine the horizontal gradient, and with two stations, minimum investigation depths are $\sim 500 \mathrm{~km}$. This technique will improve estimates of lower mantle conductivity and core size in comparison to the Apollo investigations.

A third technique, the method of magnetotellurics, provides a complete sounding at a single surface station using orthogonal horizontal components of the electric and magnetic fields [61]. This method provides spatially independent measurements with a horizontal resolution comparable to the electromagnetic skin depth.

The Farside Explorer electromagnetic sounding experiment would deploy four electrodes to distances of $\sim 10 \mathrm{~m}$ by spring launchers. This would allow 
the determination of two horizontal components of the electric field, and a fifth electrode on a mast would yield its vertical component. Three axis fluxgate and search-coil magnetometers deployed on a boom would be used to measure all three vector components of the low- and high-frequency magnetic fields, respectively. Electromagnetic cleanliness is important for this experiment, and standard techniques would be utilized to minimize spacecraft fields.

\subsubsection{Thermal evolution of the Moon}

Current knowledge In order to decipher the thermal evolution of the Moon, it is necessary to know the quantity of heat that is currently escaping its surface. The surface heat flow provides important constraints on the Moon's internal temperature and bulk composition, and is a critical measurement that any thermal evolution model must satisfy (Fig. 8). At the Apollo 15 and 17 sites, hollow fiberglass borestems were drilled to depths of approximately 1.5 and 2.5 meters, respectively, and thermal probes inserted into these measured the

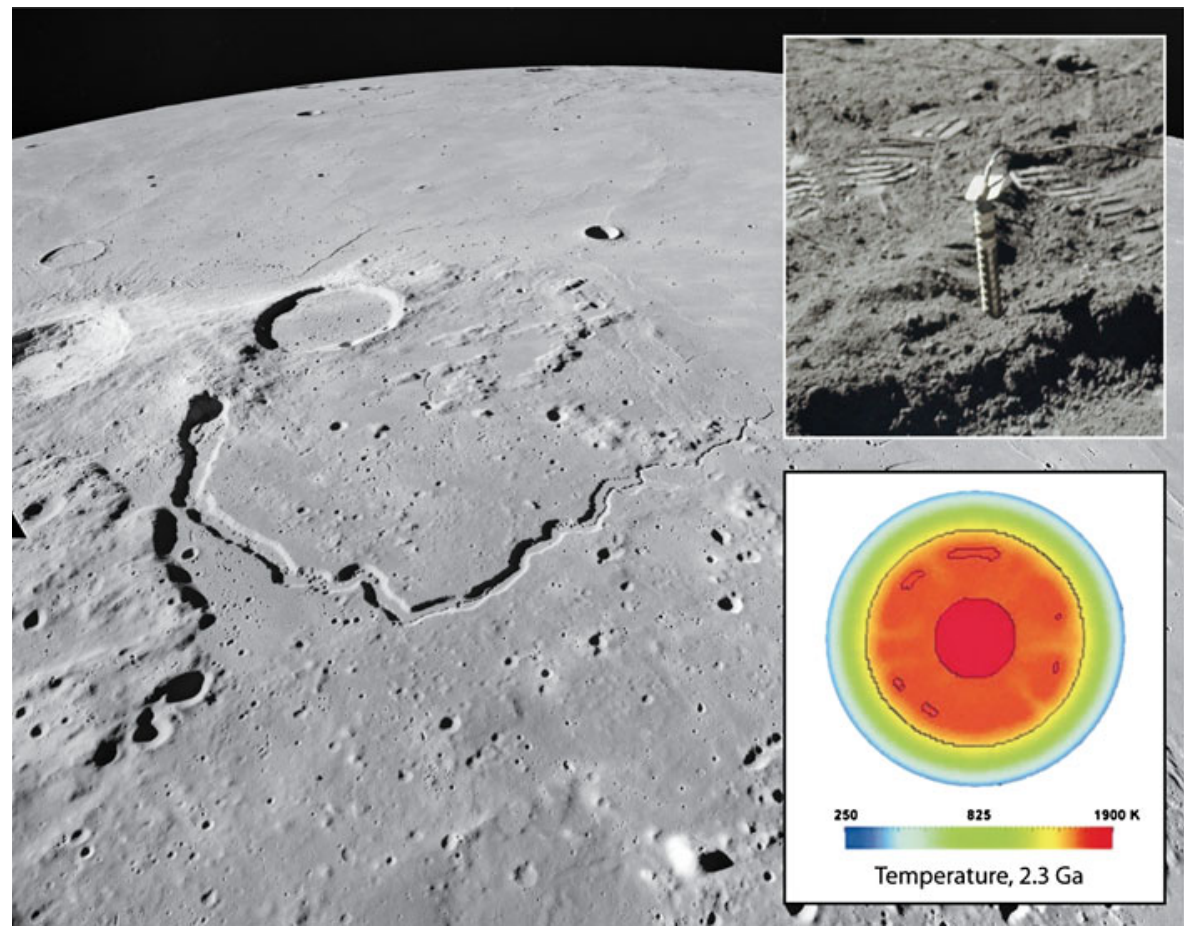

Fig. 8 The present day heat flow of the Moon is intimately related to its thermal and magmatic evolution. (foreground) Volcanic landforms in the Aristarchus plateau that formed about 2 billion years ago. (bottom right) Interior temperature of the Moon predicted from a thermal convection calculation [64] showing that lunar volcanism can occur for several billion years. (upper right) Heat flow probe at the Apollo 17 site, whose measurement is used as a constraint in thermal evolution models 
thermal conductivity and temperature at a few fixed depths. By measuring the temperature gradient and thermal conductivity, the heat flow (which is the product of the two) was reported to be 21 and $14 \mathrm{~mW} / \mathrm{m}^{2}$, with uncertainties of about $\pm 15 \%$, for the two respective sites [33].

The analysis of these data revealed several problems. First, the thermal conductivity that was estimated from the active heating experiment was found to be unreliable. The drilling of the borestems into the surface compacted and disturbed the surrounding regolith [21], and the thermal conductivity was instead estimated by measuring the attenuation with depth of the surface annual thermal wave. A second, and still unresolved, problem is that the subsurface temperatures were found to increase slowly with time. This was attributed to astronaut disturbance of the surface boundary layer, but other possibilities exist, including instrumental drift. Finally, subsequent observations from the Clementine and Lunar Prospector missions [28] demonstrated that the Moon is divided into distinct geologic terranes, with the Procellarum KREEP Terrane having much higher concentrations of radioactive elements than the adjacent Feldspathic Highlands Terrane (Fig. 3). Because both Apollo heat flow measurements were made close to the boundary of these terranes, they are unlikely to be representative of either [72].

Representative heat flow measurements from the major lunar terranes are required to constrain the abundance of radioactive elements in the lunar interior. Given the ambiguities in the Apollo heat flow results, estimates of the Moon's bulk uranium content range from an Earth-like $20 \mathrm{ppb}$ to $46 \mathrm{ppb}$. This range is too large to model confidently the Moon's thermal evolution, and precludes tests of lunar origin models.

Remaining questions Several questions remain concerning the Moon's thermal evolution:

- What is the average heat flow of the Moon?

- How does the heat flow vary among the major lunar terranes?

- What is the temperature profile of the mantle? And are portions of the mantle partially molten today?

These questions would be directly resolved by joint analyses of data obtained from the Farside Explorer heat flow probes and electromagnetic sounding experiment.

Heat flow science objectives Heat flow probes will be deployed at each landing site, reaching depths of 3 meters using a self-penetrating percussive mole device [65]. During the descent, this experiment would make temperature measurements every $20 \mathrm{~cm}$ with an accuracy of $\pm 0.05 \mathrm{~K}$, and also measure the thermal conductivity and dielectric permittivity with a $5 \%$ accuracy every $30 \mathrm{~cm}$. A major difference between the mole-based system and the Apollo heat flow experiment is that moles do not involve the drilling of a high-conductivity borestem into the lunar regolith. This minimizes the amount of disturbance 
the surrounding regolith experiences, and also removes a substantial thermal shunting effect that affected the Apollo data.

To realize the science objectives, the heat flow must be determined with an accuracy better than $15 \%$, and to achieve this, both the thermal gradient and thermal conductivity must be determined to better than $10 \%$. Thermal conductivity can also be estimated from the thermal diffusivity (assuming the density and heat capacity are known), and this would be measured in the active heating mode during mole descent, and from the propagation of the annual thermal wave when the instrument was in its monitoring mode. These diffusivity methods would perform a consistency check with the active-heating thermal conductivity experiment.

\subsubsection{Surface composition}

Current knowledge The samples returned by the Apollo and Luna missions are well documented and have provided a wealth of data from a few known locations on the surface. The global composition of the lunar surface has been assessed from orbiters using UV-VIS spectroscopy for iron and titanium, and gamma-ray spectroscopy for a few major and minor elements. Furthermore, spectroscopic techniques have been used to place constraints on the global mineralogy of the lunar crust. Combined, these datasets attest to the diversity of rock types that were formed during the initial differentiation and subsequent magmatic activity of the Moon. Importantly, these data demonstrate that there are rock types in both the highlands and South Pole-Aitken basin that are not represented in the Apollo and Luna sample collections [41, 55].

Remaining questions It is a challenging task to obtain absolute elemental abundances and mineralogy from remotely sensed data. Such measurement techniques are often calibrated by ground truth measurements, and the farside highlands and South Pole-Aitken basin are sufficiently different from the Apollo samples as to call into question the validity of some remote compositional estimates for these regions [41]. It is not possible to measure the abundance of many trace elements from orbit, and this has left unresolved many questions concerning the magmatic evolution of the Moon. A better characterization of crustal composition is required to constrain the bulk composition of the Moon, and key unknowns are the composition of the farside highlands and the lower crust. The origin of materials in the giant South PoleAitken basin are unknown, with possibilities including pristine lower crustal materials, a differentiated impact melt sheet, or a mixture of crustal and mantle materials [73].

Surface chemistry science objectives Several techniques can be used to measure in situ absolute elemental abundances, such as laser-induced breakdown spectroscopy or particle-induced X-ray spectrometry. In situ passive neutron/gamma-ray spectrometry [14] is here advocated as this instrument would benefit enormously from the long duration of the Farside Explorer 
mission. Though similar measurements have been made previously from orbit, the long accumulated measurement times for these surface measurements at a single locale would reduce the statistical uncertainties for elemental concentrations by orders of magnitude compared to those obtainable from orbit. A surface neutron/gamma-ray spectrometer would make bulk elemental abundance measurements in a half sphere with a radius of $\sim 50-100 \mathrm{~cm}$ around the instrument, whereas other instruments would only investigate the top microns to $\mathrm{mm}$ of the surface. These measurements would provide for a more robust calibration of remote sensing data by making important ground truth measurements in two compositionally unique terranes.

Gamma-ray spectroscopy can measure the elemental concentrations of many major and trace elements, such as iron, titanium, silicon, calcium, aluminum, thorium, potassium, and uranium [58]. Neutron spectroscopy is complementary to gamma-ray spectroscopy and is highly sensitive to the concentrations hydrogen and neutron absorbing elements. The most important neutron absorbing elements in lunar soils are the major elements iron and titanium and the trace elements gadolinium and samarium. Gadolinium and samarium are important for understanding many details of lunar differentiation and evolution, and titanium is an important indicator of the presence of solar wind implanted gases, such as ${ }^{3} \mathrm{He}$.

\subsubsection{Earth-Moon impact hazards}

Current knowledge Asteroids and comets are derived from beyond the orbit of Mars and occasionally they encounter the Earth. The vast majority of these meteoroids is small, and burn up in the atmosphere, but a smaller number of larger objects pass through the atmosphere to make an impact crater on the surface. Such events can be catastrophic to life, either locally or globally. Two primary objectives of investigating impact hazards are to determine the sizefrequency distribution of these objects, and to determine the probability that they will collide with the Earth.

The Moon is subjected to the same flux of impactors as the Earth, but unlike the Earth, where small bodies are destroyed in the atmosphere before hitting the ground and where impact structures are rapidly eroded, its surface is an excellent recorder of collisions with objects at all sizes. The present flux of impactors gives rise to new impact craters that can be imaged from orbit, and the impact itself produces a visible and thermal emission (an impact flash) that is detectable from Earth or lunar orbit (Fig. 9). Observation of the Moon's surface thus provides a complementary approach to understanding the flux and size distribution of objects in near-Earth space, as well as providing known seismic sources for investigations of the lunar interior.

Impact hazard studies have traditionally made use of the detonations of meteors in Earth's atmosphere and telescopic surveys of larger near-Earth objects. The telescopic survey of near-Earth objects is now almost complete for objects with diameters greater than about $1 \mathrm{~km}$. These observations constrain the impact rates on the Earth and Moon, and predict significant temporal 


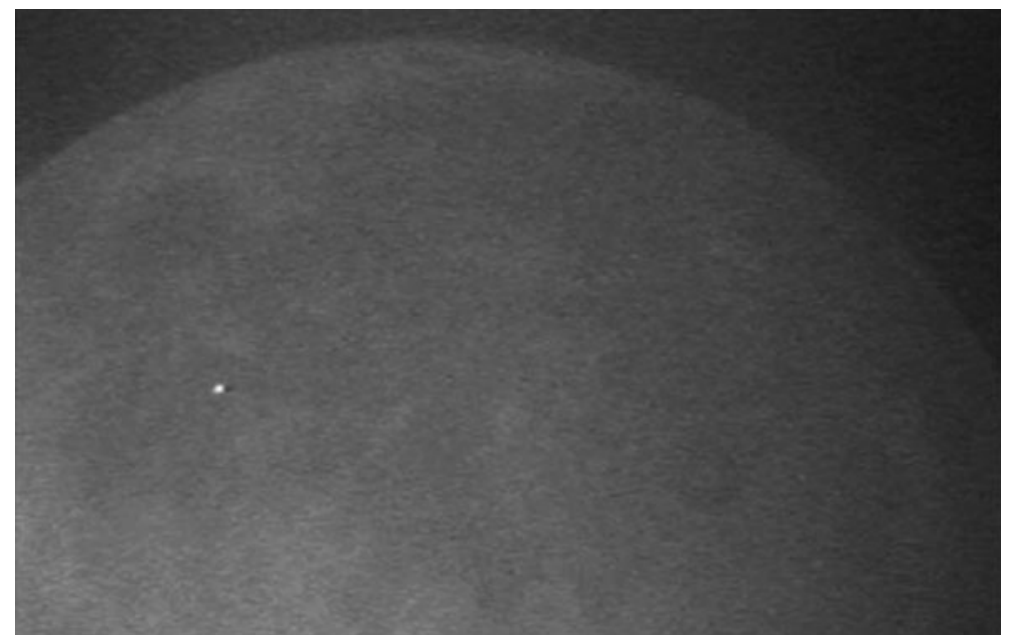

Fig. 9 An impact flash detected from one of the telescopes at Huetor-Santillàn. The Moon is illuminated only by Earthshine. Image from [51]

variations in their impact flux, either as a time-of-day phenomenon for the Earth or solar phase for the Moon [35, 36, 39]. The Moon's synchronous rotation also gives rise to a time-averaged impact flux that is greater on its western hemisphere than its eastern hemisphere by about $20 \%$ [35]. Though predicted by theory, the magnitude of these temporal and spatial variations is difficult to measure in practice.

Satellites in orbit about the Earth have recorded the frequency of light bursts that occur during the atmospheric entry of meteoroids that are less than 10 meters in diameter, and these can be converted into masses using the luminous efficiency parameter, which empirically relates the light emitted in the visible to the kinetic energy of the meteoroid [3, 52, 76]. Camera surveys have extended these estimates down to $10 \mathrm{~cm}$ and radar echoes have allowed the mapping of radiant distributions for bolides down to the $\mathrm{mm}$ size range [11].

A record of the near-Earth meteoroid flux is also available from the Apollo seismic network that detected about 1700 impacts with masses ranging from $\sim 0.1$ to $100 \mathrm{~kg}$ during 8 years of operations $[48,49]$. Video observations have confirmed that these lunar meteoroid impacts are observable from Earth via the light they emit during impact [51], and impact-monitoring programs [66] have since detected more than 200 impact flashes.

The radiant distribution at Earth calculated from near-Earth object orbitalelement models is in broad agreement with the radar-inferred distribution from meteors [39]. Presuming that the orbits of the smaller meteoroids mimic those of the well characterized $\mathrm{km}$-sized objects, it is possible to estimate the impact flux on the Moon at all sizes. These estimates, however, appear to be three times smaller than those derived from lunar impact flash data [51]. 
Seismic modeling of meteoroid impacts with the Moon and the Apollo seismic data [39], in contrast, appear to favor the high impact rates derived from impact flashes.

Finally, estimated impact rates can be compared to the size-frequency distribution of small lunar craters using impact crater scaling laws. The meteoroid flux estimated from the detonation of bolides in the terrestrial atmosphere [9] is predicted to produce craters on the Moon that are 10-100 meters in diameter, and the measured number of craters favors the lower impact rate derived from orbital-element models instead of the higher estimates from the impact flash data. If the young lunar cratering record were found to be consistent with the rates of bolide detonations in Earth's atmosphere, this would imply that most small lunar craters are primary craters, and not secondaries. Secondary craters are formed from the material ejected from a primary crater and are a significant source of controversy in the crater chronology literature.

Remaining questions The impact fluxes for bolides in the size range of $1 \mathrm{~cm}$ to $1 \mathrm{~m}$ are uncertain by a factor of at least 3 . Recent impact rates deduced from crater counts on the youngest units sampled by Apollo [26] are consistent with atmospheric detonation estimates, but this modeling procedure involves several parameters that are imperfectly known, such as the optical burst efficiency and impact crater scaling law exponents (Fig. 10). Impact rates derived from lunar impact flashes are at least three times larger, but these estimates depend upon the luminous efficiency parameter, which is poorly known and which varies among meteoroid classes. If differences in the impact rates determined from crater counts, bolide detonations and impact flashes exist, this could reflect long- or short-term temporal variations in the delivery of asteroidal materials to the Earth-Moon system. Alternatively, it is possible that the neglect of time-of-day variations in the terrestrial impact rates, and the neglect of lunar phase variations in the lunar rates, could have biased these estimates.

Several questions remain unresolved concerning the impact flux in the nearEarth neighborhood:

- What is the present impact flux of bolides in the size range of centimeters to meters?

- What is the size-frequency distribution of small objects colliding with the Earth and Moon? And what is their velocity distribution?

- What is the magnitude of temporal and spatial variations in the lunar impact rates?

These questions would be resolved by joint analyses of data from the Farside Explorer dedicated impact flash monitoring camera and surface seismology experiments.

Impact monitoring science goals Farside Explorer would continuously monitor the lunar meteoroid flux from the vantage point of the Earth-Moon L2 Lagrange point. These observations would be ideal because there is a complete 


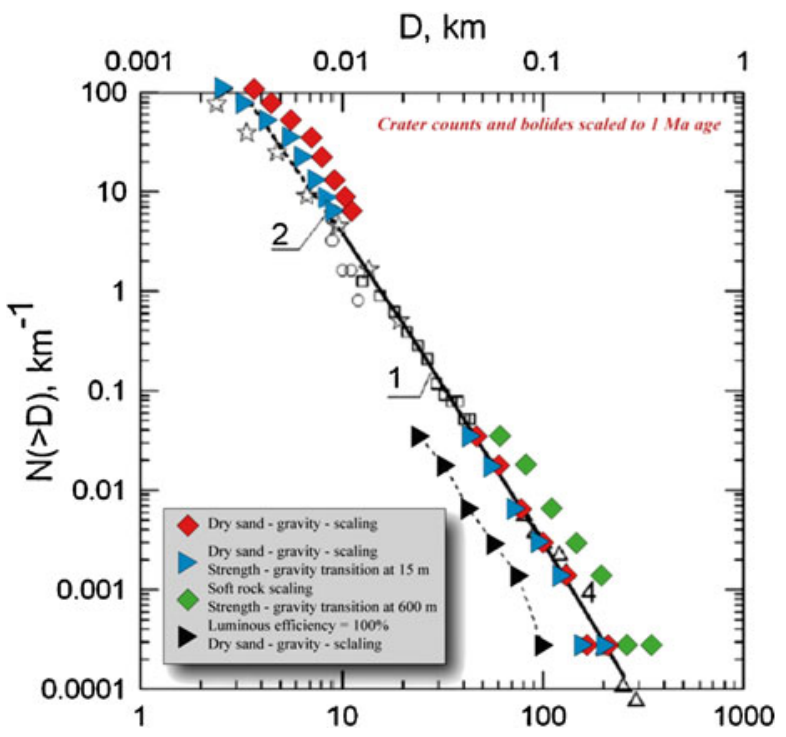

Fig. 10 Observed and estimated lunar impact cratering rates as a function of crater diameter. Lunar crater counts for a 100 million year old surface are plotted as open symbols, whereas estimates based on bolide detonations in Earth's atmosphere are plotted as filled diamonds and triangles. Estimated crater sizes use several hypothesis: Red diamonds assume gravity scaling of impact crater formation, blue triangles use strength scaling for sand-like targets, green squares use strength-scaling for soft rock targets, and black triangles correspond to a $100 \%$ luminous efficiency and gravity scaling. Considerable uncertainty exists in the present cratering rate, especially below $100 \mathrm{~m}$, which corresponding to bolides smaller than $10 \mathrm{~m}$. Image redrawn after [26]

lack of Earthshine illuminating the unlit portions of the Moon's farside hemisphere, and the lack of an intervening atmosphere would allow for infrared measurements. Combined with the proximity of L2 to the lunar surface, these observations would allow for the detection of much smaller impacts than could be accomplished from a comparable terrestrial observatory. From these measurements, a more precise picture of impact processes in the Earth-Moon neighborhood will be obtained.

Considering the expected detections of several hundreds of events per year, the main goals of the impact monitoring program are to: (1) determine seismic source locations and times for the seismology experiment, (2) obtain the average impact rate on the Moon and map any spatial and temporal variations that might exist, and (3) determine the optical-magnitude impactfrequency relationship. Combining the measured optical magnitudes with simultaneous data from the seismology experiment on the surface offers a unique opportunity to explore the partitioning of impactor kinetic energy into seismic and thermal energy at scales that are impossible to reach in laboratory experiments [47].

Monitoring the temporal variations of the impact flux over the lunar month would provide constraints on the meteoroid sources, since these variations are 
directly related to the radiant distribution of impactors entering the EarthMoon system. Comparison with estimates based on orbital-element models and encounter probability calculations would improve our understanding of the fate of meteoroids once they separate and evolve from their parent bodies.

Impact monitoring science requirements The main technical specifications of the impact flash monitoring camera should include a high frame rate, a field of view covering the entire lunar disk, and a real-time processing for downloading only those images with positive detections [50]. For the seismic investigations, the spatial resolution and absolute time recording should be better than $1 \mathrm{~km}$ and $0.5 \mathrm{~s}$, respectively.

Multispectral observations are essential in order to monitor the thermal evolution of the impact site and ejecta cloud. Ideally, at least two bands would be utilized, one in the visible from $0.4-0.8 \mu \mathrm{m}$, corresponding to peak emissions covering the vapor phase, and a second in the near infrared from 1-2.5 $\mu \mathrm{m}$, corresponding to peak emissions for silicate melts present in the ejecta. In combination with simultaneously acquired seismic data, repeated monitoring of the thermal evolution of impact flashes will help refine the relationships between emitted light, impactor mass, and impact velocity. In a few rare cases, the impact velocity may be determined directly as the bolide passes through the camera's field of view. This work will stimulate progress in impact cratering mechanics by offering natural experiments at scales far larger than those achievable in the laboratory.

Farside Explorer would monitor lunar impact flashes on the farside of the Moon, and a complete view of the lunar impact rate would be obtained by using ground-based observations of the nearside from Earth. These observations are currently being conducted with a small number of mid-sized telescopes, and an international network for the detection of impact flashes (ILIAD) is under construction with an expected completion date in 2014.

\section{Synergy with other missions and programs}

In addition to being a stand-alone mission that would address first-rate scientific questions, Farside Explorer would also complement past and future missions by providing much needed ground truth measurements from the surface of the Moon. Crustal thickness determinations made beneath the two landers would be invaluable for the construction of a global high-resolution crustal thickness model using data from NASA's lunar gravity mapping mission GRAIL. The interpretation of NASA's Lunar Reconnaissance Orbiter Diviner radiometer data would benefit enormously from the Farside Explorer subsurface temperature profiles. The United States planetary science decadal survey has identified a South Pole-Aitken sample return mission as one of its top priorities in the next decade [13], and the in situ surface and subsurface measurements made in this basin by one of the Farside Explorer landers would 
provide critical measurements for constraining the origin and evolution of these samples.

Farside Explorer would also provide valuable radio-astronomy measurements that would extend the frequency range of those being made from terrestrial low-frequency arrays. The overlapping frequency range between Farside Explorer and the large European ground-based array LOFAR, the Ukrainian low-frequency array UTR2 [32] and the Nançay decameter array [6] would allow for extensive comparisons, cross-calibrations of intensity and polarization, evaluation of the Earth's ionospheric absorption, and VLBI interferometric measurements.

The Farside Explorer impact monitoring of the Moon's farside hemisphere would perfectly complement monitoring programs of the lunar nearside made from observers on Earth. These terrestrial observations are currently being conducted by several groups, such as through the International Lunar Impact Astronomical Detection network (ILIAD), and by combining the orbital and terrestrial datasets, a complete view of the lunar impact rate would be obtained.

Finally, the United States planetary science decadal survey has identified a lunar geophysical network as one of seven priority missions for possible implementation in NASA's New Frontiers program. The launch date of this mission would be close to 2026, and this would coincide approximately with the launch of ESA's fourth Cosmic Vision medium-class mission. A NASA-led geophysical network would provide no more than 4 geophysical stations, with most, if not all, being situated on the lunar nearside hemisphere. In combination with an ESA-led mission to the farside, international cooperation would allow for a unique opportunity by offering a much more robust geophysical network than could be afforded by any single agency. Other countries have expressed interest in placing landers on the Moon, and if these contained geophysical instrumentation (such as is planned for the Japanese SELENE2 mission concept), these stations would contribute to an already operating geophysical network [75].

\section{Mission profile}

The Farside Explorer space segment includes two spacecraft to land on the farside of the Moon, an instrumented relay satellite, and the launcher (either a Soyuz-Fregat or Ariane 5 shared commercial launch). The proposed mission concept is innovative by using a halo orbit about the Earth-Moon L2 Lagrange point (LL2) to provide a communications relay to the farside landers while simultaneously enabling the impact flash monitoring program.

Two options are possible for the launch segment. The first strategy uses a Soyuz-Fregat, whereas the second strategy uses a shared Ariane 5 geosynchronous transfer orbit (GTO) launch together with a commercial spacecraft (see Fig. 11 for accommodation outlines). The second option allows a significant mass increase for the spacecraft composite, but does not increase significantly 
Fig. 11 Soyuz-Fregat (left) and Ariane 5 (right) spacecraft accommodations. Image courtesy of Astrium Satellites
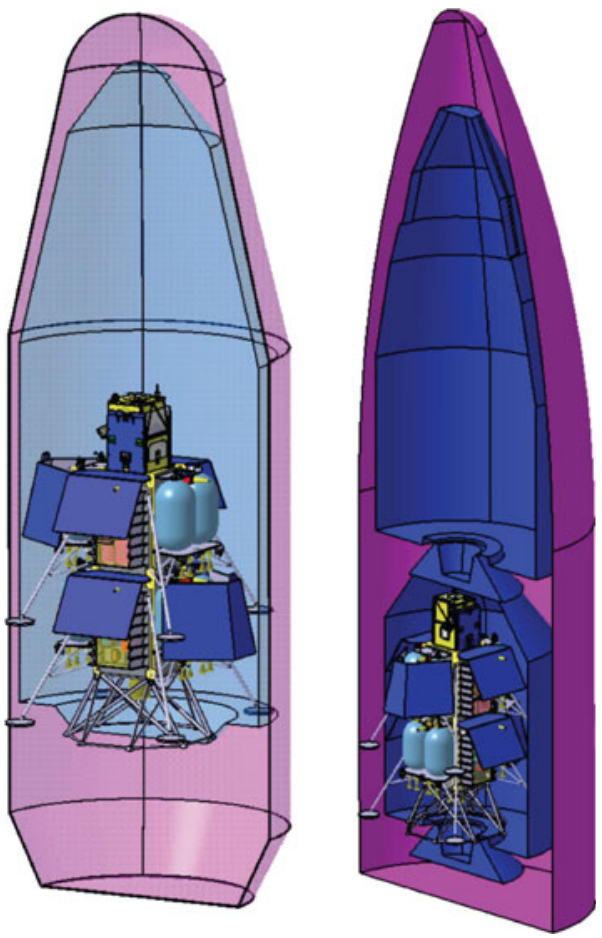

the cost since the launch provider charges a price per $\mathrm{kg}$ for GTO launches, regardless of the launcher type.

As previous studies using Soyuz launchers have proven to be marginal in mass (such as the Astrium Satellites "MoonTwins" study), we have not favored a direct injection into a ballistic trajectory to LL2 or a trans-lunar injection. We instead propose a longer, but more mass-efficient, launch sequence where the spacecraft is first injected into a geosynchronous transfer orbit and then waits for the proper configuration to fly along a weak stability boundary (WSB) trajectory that goes first to the Earth Sun L1 Lagrange point (EL1) and then to the Earth-Moon L2 (see Fig. 12) [1, 53, 54]. A GTO waiting orbit offers several advantages over a direct trajectory: it allows several launch windows per month with a similar $\Delta \mathrm{V}$, it requires less post-launch $\Delta \mathrm{V}$, and it provides a longer cruise time for calibrations, readiness tests, and flight system performance. Following insertion into GTO, two staging options are considered:

1. Each spacecraft includes the propellant for the rest of the mission (transfer to EL1, LL2 and landing for the probes; transfer to EL1 and LL2 for the relay satellite).

2. The upper lander and relay satellite are bound together during the EarthMoon cruise as a composite (baseline). After LL2 halo orbit injection, the satellite and lander are separated. 
Fig. 12 Low $\Delta V$ trajectory to the Moon. After insertion in a geosynchronous transfer orbit, the two spacecraft are injected into a 150-day WSB trajectory along the

Earth-Sun L1 gravity manifold. This trajectory returns to the vicinity of the Moon, allowing insertion into the Earth-Moon L2 halo orbit. Modified after [53]

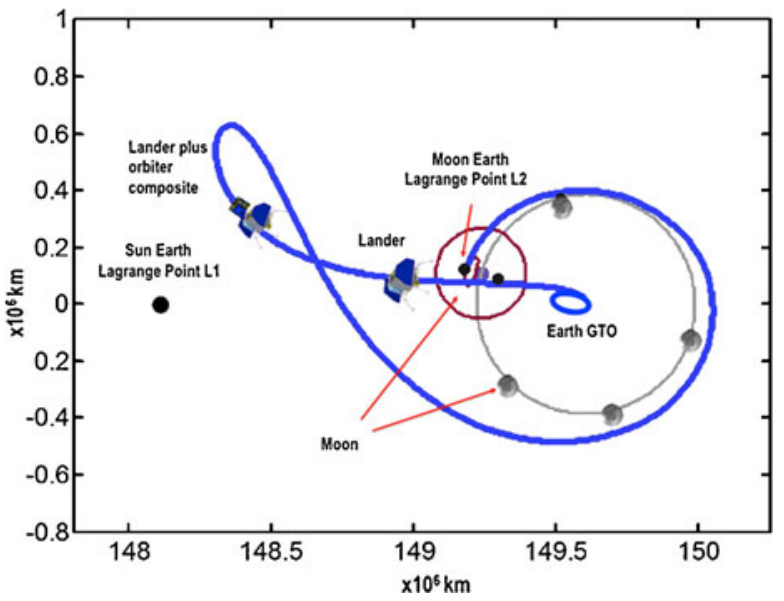

The second strategy allows for a very simple bus for the relay satellite as it does not require a propulsion stage (see Table 2 for a comparison of the two approaches).

From GTO, the lunar probes are injected one after another into a 150-day ballistic trajectory along the EL1 gravity manifold through two or three perigee burns. The proposed trajectory uses the instability of the manifold next to EL1 to return to the vicinity of the Moon. At lunar arrival, the intersection of the manifolds of the Earth-Sun and Earth-Moon system allows for the insertion, at a very low $\Delta \mathrm{V}$, of the mission elements into an Earth-Moon LL2 halo orbit. The LL2 halo orbit will be the quasi-final orbit for the relay satellite, but only a waiting orbit for the two landers. The spacecraft LL2 waiting orbit allows the access to any location on the lunar surface with a single large maneuver (the braking burn). The landing strategy is to follow the unstable WSB down to the surface and to set to zero the relative speed with respect to the landing site. Ground control would monitor constantly the spacecraft landing through the relay satellite. As a result of the waiting orbit strategy, the control of only one probe at a time is required for the critical landing phase.

Farside Explorer is a low risk mission: The use of a GTO allows safe waiting for the proper ephemeris configuration; the use of a GTO intermediate orbit allows for the possibility of a shared Ariane 5 commercial launch; the WSB trajectory provides a significant spacecraft mass increase with respect to other transfer options; the LL2 $\Delta \mathrm{V}$ orbit injection cost is very low; and only one spacecraft at a time encounters critical phases, allowing for a more secure operation strategy.

\subsection{Operational halo orbit}

The chosen operational halo orbit results from a compromise between several mission requirements: use for a relay satellite with almost constant visibility of the farside landing sites from Earth [22], implementation of the lunar impact 


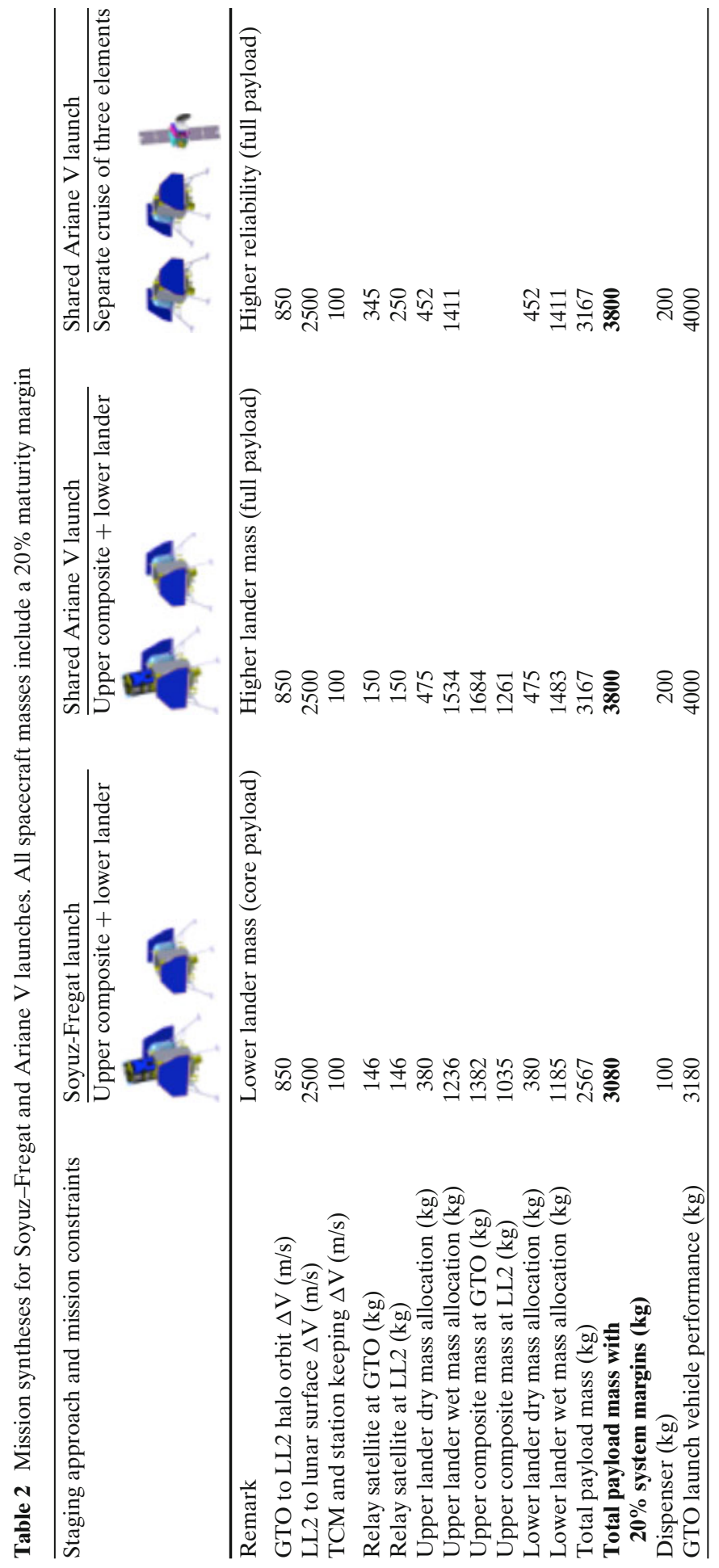


flash and magnetic field payloads, minimization of maintenance requirements, and low cost injection from the transfer orbit. Several LL2 halo orbits are possible, and for operational reasons, a 2:1 resonant orbit (14 days period, see Fig. 13) is preferred with altitudes that vary between about 30,000 and $60,000 \mathrm{~km}$. For this pseudo-orbit, the relay satellite would be in constant visibility of the equatorial lander and would be in visibility of the southern lander $90 \%$ of the time. A slightly higher altitude orbit is possible that would enable a constant visibility of the two landers.

\subsection{Landing phase}

The landing phase starts from the de-orbit burn to the final stationing of the probe on the lunar surface. Two strategies can be considered: a simple strategy similar to a Hohmann transfer, or a weak boundary transfer to a low altitude (baseline). In both cases, a final burn is required to align the state vector of the probe to the landing dynamic state (Fig. 14). As there are no precise landing requirements, hazard avoidance can be made on the basis of a passive approach (target within safe zone) with attitude acquisition by Doppler radar. The first landing is foreseen at dawn of the landing site, allowing two weeks to deploy the science instruments before lunar night. Landing of the second lander occurs one month (one lunar day) later.

In order to simplify the probe guidance, navigation and control (GNC), no precision landing capabilities are foreseen. The American and Soviet space

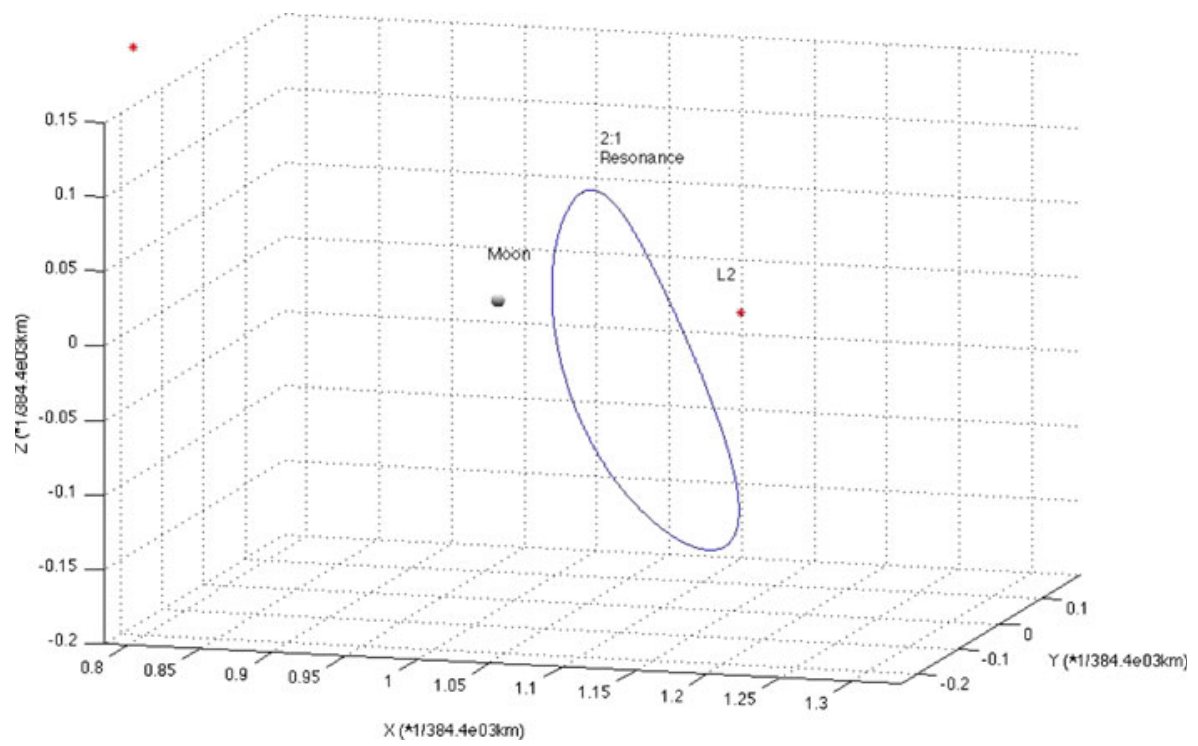

Fig. 13 Operational halo orbit. This 2:1 resonant pseudo-orbit has a 14-day period and has altitudes that vary from 30,000 to $60,000 \mathrm{~km}$ above the lunar surface. Axes are scaled by the mean Earth-Moon separation 


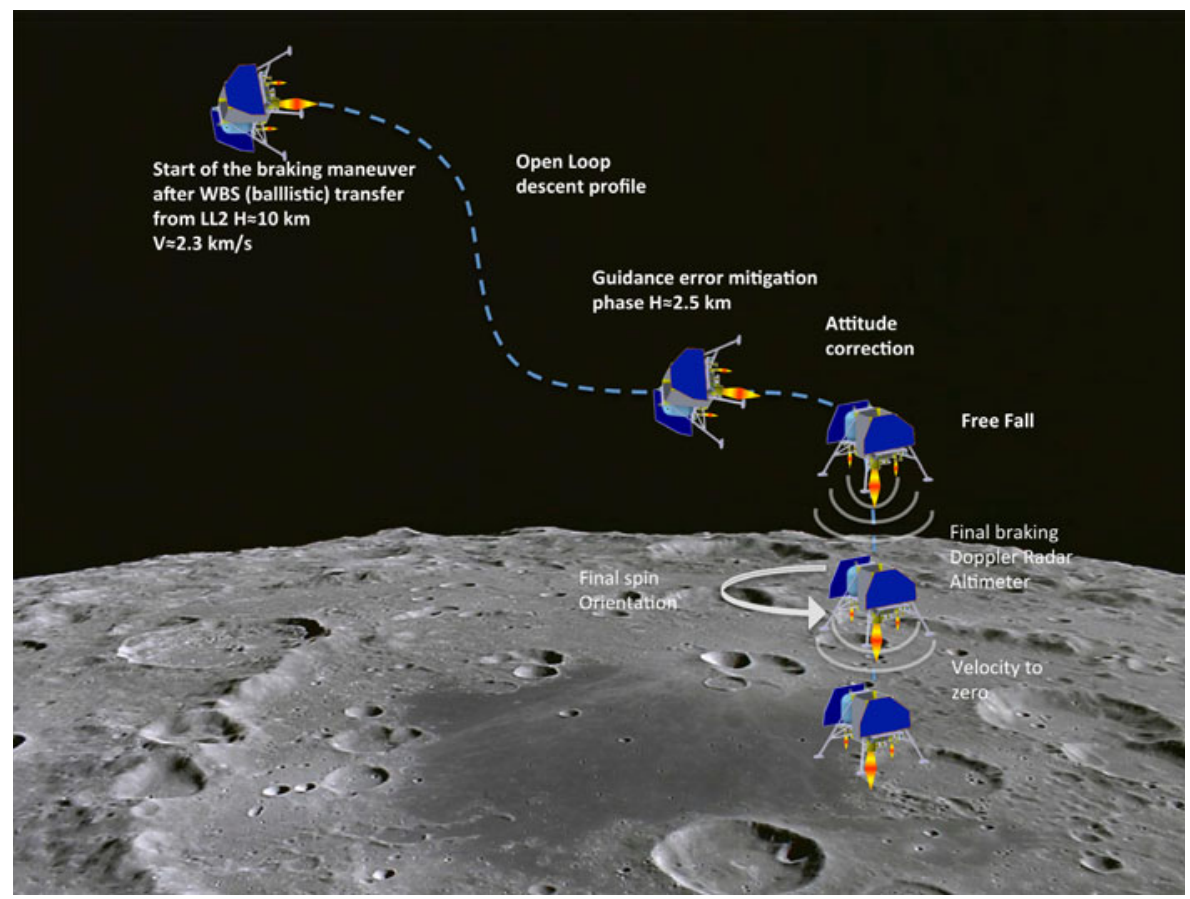

Fig. 14 A possible landing phase outline

programs have demonstrated that precision landing and hazard avoidance capabilities are not required to safely land a robotic spacecraft on the Moon. Whereas all previous robotic landings were performed with little detailed information about the lunar surface, site selection for Farside Explorer will mitigate against potential surface hazards by using existing high-resolution images of the surface, slope maps from high precision laser altimetry and stereo images, and rock abundances from orbital radar and radiometer data.

\subsection{Communication and ground segment requirements}

The proposed communications and ground segment baseline is to use ESA's network of ground stations (Redu, Vilspa, Kourou, Perth) during launch and early operations (LEOP) and landing, and NASA's stations in case of contingency. A reduced number of stations would be baselined during the ballistic transfer to LL2, while for critical phases, the full network might be reserved. Communications between the relay satellite and landers is foreseen during daylight at each lander's location in either UHF (low rate) or X band (high rate). Preliminary estimates of the UHF return link allows for $256 \mathrm{kbps}$. Due to the high data rates, $\mathrm{X}$ band is preferred for the telecommunication link between the relay satellite and Earth. Mission operations and control will be 
performed at ESOC, while the data will be stored and processed at ESRIN or at any dedicated national facility. Several user centers at the instrument PI's institutions would process the scientific products.

\subsection{Critical issues}

Single lander mission The scientific return from a single-lander mission (either due to a lander failure or descope) would be diminished by more than a factor of 2. For the radio astronomy experiment, the lack of a second station would preclude source localization techniques. New deep moonquake nests could not be located with a single station, and the known deep moonquake nests might not be uniquely identified. Although core detection could be accomplished, the core size would not be determined. Crustal thickness beneath the lander would be obtained, but tomography of the upper mantle would be difficult. For the heat flow experiment, only one lunar terrane would be investigated instead of two. For the electromagnetic sounding experiment, it would not be possible to use the geomagnetic depth sounding technique to investigate the lower mantle.

Relay satellite failure In the case where the relay satellite fails before landing of the lunar probes, the landing sites could be repositioned to the nearside, allowing for direct communication with Earth. Nearside landing sites would not be protected from terrestrial radio-frequency interference, but the geophysical investigations would be able to achieve many of the International Lunar Network objectives [71].

\section{Model payload}

The Farside Explorer mission consists of two essential components: an instrumented relay satellite to be inserted into a halo orbit about the EarthMoon L2 Lagrange point, and two identical spacecraft that make soft landings on the lunar surface. The two landers would contain a suite of state-ofthe-art instrumentation: a radio astronomy receiver, long- and short-period seismometers, a heat flow probe, an electromagnetic sounder, surface cameras, and a geochemical experiment. A robotic arm is included on each lander for deployment of the seismometer and heat flow experiment, but lighter alternatives with fewer degrees of freedom are also available. The relay satellite would contain an impact flash camera, and a magnetometer. Radio astronomy interferometric correlations could be performed on the orbiter in order to reduce the amount of data transmitted to Earth.

The surface and orbital experiments are synergistic (Fig. 15). The times and locations of impact events on the lunar surface obtained from the orbiting camera would be used as known sources for the seismic experiment. By monitoring the magnetic fields on the surface of the Moon and from orbit, it 


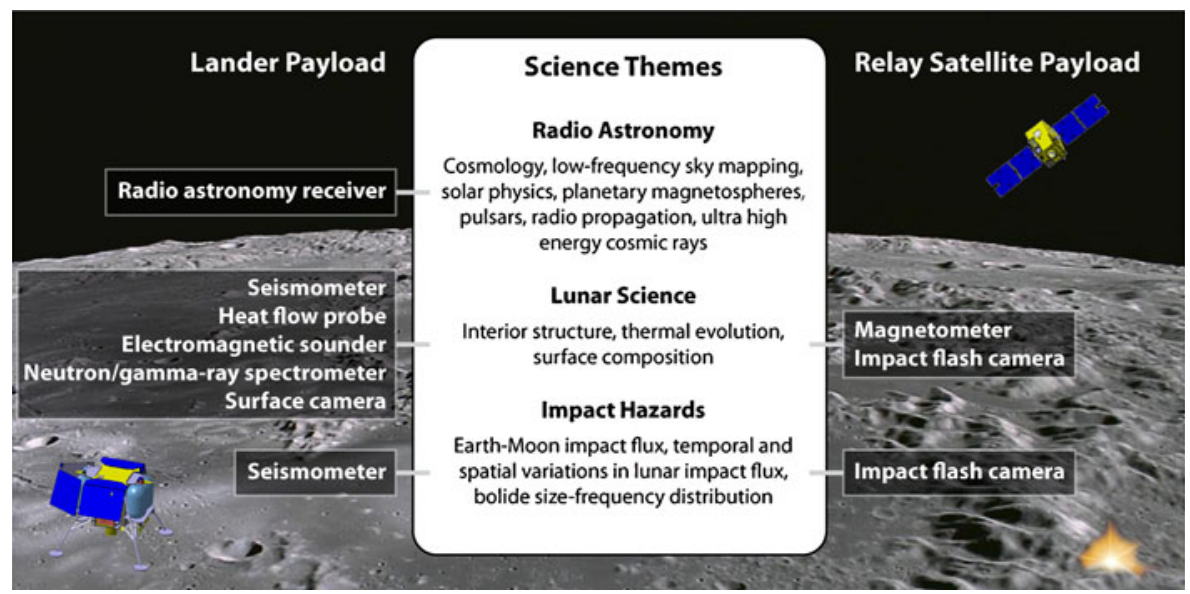

Fig. 15 Relationship of Farside Explorer science themes to the lander and orbiter payload. Lunar science and impact hazard objectives are addressed by synergistic measurements from the surface and orbit

would be possible to determine an electrical conductivity profile of the Moon from the crust to core.

The amount of mass and power available for the payload of a small lander powered by solar panels and batteries is limited, especially during the night. Though several possible instruments exist that could fulfill the primary science objectives, a specific set with known resource requirements were evaluated to demonstrate the feasibility of the mission concept. Based on the properties of these instruments, two versions of the lander payload are presented: a core science version, whose resources are compatible with a Soyuz-Fregat launch, and a full science version that is compatible with a shared Ariane 5 launch (Table 3). The resources of the relay satellite are less critical.

The core version of the model payload fulfills all of the primary science objectives. The full science version has a second heat flow experiment on each lander, two additional horizontal short-period seismometers, a geochemistry instrument, a high-frequency search coil magnetometer, and a vertical mast for the radio astronomy and electromagnetic sounding experiments. To reduce mass, the radio astronomy and electromagnetic sounding experiments share common antennas.

\subsection{Radio Astronomy Receiver (RAR)}

The radio astronomy experiment includes two or three electric dipoles per lander connected to a radio astronomy receiver that performs quasi-continuous spectral analysis and waveform capture snapshots in the low frequency range from $\sim 0.016$ to $40 \mathrm{MHz}$. Full description of the signal from a compact radio source requires 7 parameters (total flux, linear and circular polarizations, 
Table 3 Lander and relay satellite payload masses, power and data rates

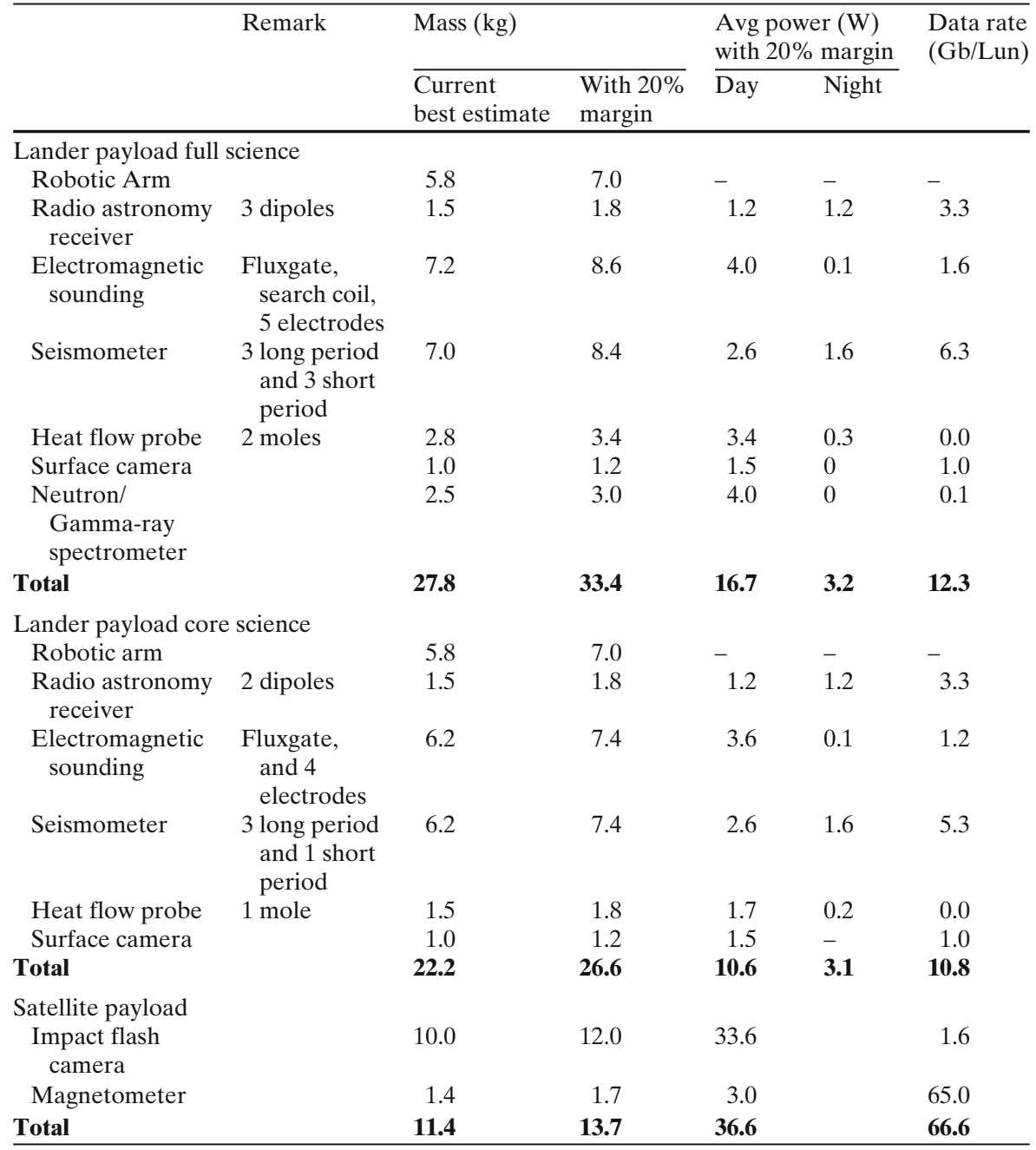

For the lander payload, two versions are given, a core science version that meets the primary science objectives, and an enhanced full science version

source direction, and angular source size). A system of 2 dipoles connected to a receiver allows the measurement of 4 instantaneous quantities (signal auto- and cross-correlations), whereas with 3 dipoles, 9 quasi-instantaneous quantities can be measured ( 3 auto- and 3 complex cross-correlations) allowing full description of the wave.

Waveform snapshots recorded simultaneously at two locations can be crosscorrelated to compute the complex visibility corresponding to the lander baseline, or its projection perpendicular to the source direction. Measured time shifts between the two waveforms allow the selection of source directions 
along circles on the celestial sphere prior to correlation. The interferometric measurements will constrain the instantaneous angular extent of strong radio sources along the baseline direction, and will be used to build average lowfrequency radio sky maps by global inversion of long time series of visibilities.

Instrument characteristics The radio astronomy receiver being built by LESIA (France) meets the scientific objectives of $\sim 10 \mathrm{kHz}$ spectral resolution at the lowest frequencies and $100-200 \mathrm{kHz}$ at higher frequencies. A radio spectrometer (RS) has been developed that combines a multi-channel receiver with direct conversion below about $1 \mathrm{MHz}$ with a superheterodyne sweptfrequency analyzer above $\sim 1 \mathrm{MHz}$, and a time domain sampler (TDS) for waveform capture. In the RS receiver, wavelet-like transform and channel auto- and cross-correlations provide flux and polarization spectra that are compressed and sent to the digital processing unit. The TDS samples voltages from the dipoles at 20-50 Msamples/sec, and it is possible to combine the TDS with the RS in order to digitize a limited spectral band at a reduced sampling rate. The digitized waveform can be processed locally for event detection, synchronous and de-dispersion, or sent to the relay satellite.

Performance assessment With a typical receiver noise of $7 \mathrm{nV} \mathrm{Hz}^{-1 / 2}$, the skylimited sensitivity regime is reached above $1 \mathrm{MHz}$ for monopole lengths of $\sim 2 \mathrm{~m}$, while lengths of 5-10 m increase the sensitivity below $\sim 500 \mathrm{kHz}$. A separation of $\sim 1700 \mathrm{~km}$ between landers will allow a maximum interferometric angular resolution of about 1' at $1 \mathrm{MHz}$ for discrete sources, but sky images synthesized via the inversion of long series of measurements will have a coarser resolution.

Instrument resources The radio astronomy receiver mass is $1.5 \mathrm{~kg}$. Antennas are to be shared with the electromagnetic sounding (EMS) experiment (Fig. 16) and power usage will be $1 \mathrm{~W}$ on average, and $2.5 \mathrm{~W}$ peak. The receiver size is $21 \mathrm{~cm} \times 30 \mathrm{~cm} \times 2.5 \mathrm{~cm}$. If the EMS antennas were not available, an additional 3-4 kg would be required for the two horizontal dipoles and vertical mast. The RS data rate can be adjusted between 180 bps and 730 bps, and onboard lossless compression allows a reduction by a factor of about 1.3. Further reduction can be obtained by a $<100 \%$ duty cycle. Waveform capture produces a raw data rate of $3-60 \times 10^{7}$ bps during brief snapshots timed to be compatible with the transfer rate to the relay satellite and/or storage capability.

Specific interface requirements Electromagnetic cleanliness is an important requirement for sensitive and accurate radio measurements, and the orientation of the antennas should be known a posteriori with an accuracy $\leq 1 \mathrm{~m}$ and $1^{\circ}$. During interferometric measurements, accurate relative timing between the two landers is required. A central processor on the relay satellite is desirable to synchronize and correlate the data streams in order to obtain the complex visibility function of an observed celestial source. 
Fig. 16 The radio astronomy receiver (RAR) and electromagnetic sounding (EMS) experiment utilize the same antennas to measure all three components of the high- and low- frequency electric fields, respectively. RAR measures differences in voltage of the separate antennas, whereas EMS measures differences in voltage between the antenna end points

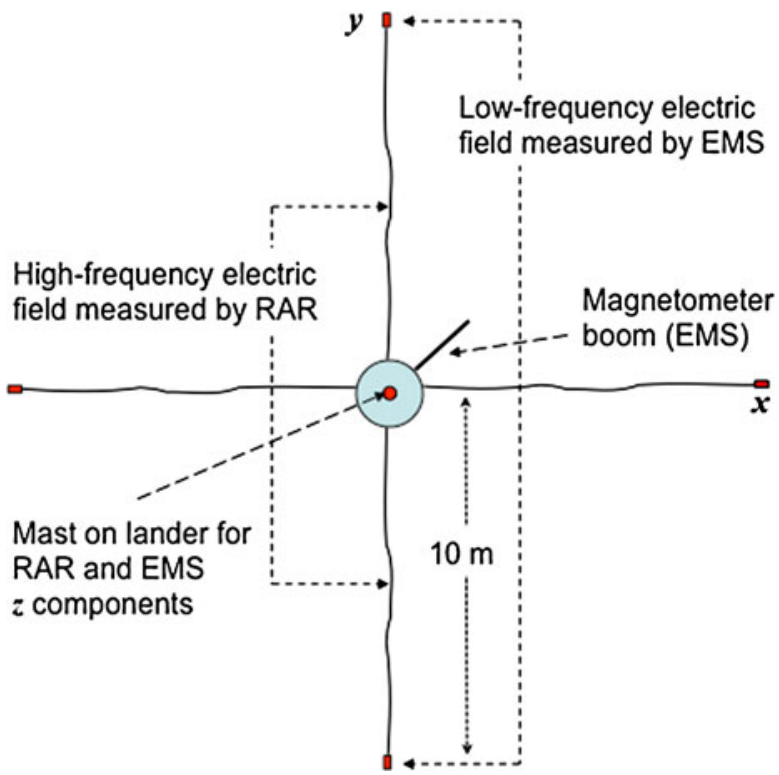

\subsection{Electromagnetic sounder (EMS)}

The electromagnetic sounding experiment uses high-heritage electric and magnetic field sensors and is here based on the instrument in development by the Southwest Research Institute (US) and the University of California, Berkeley (US). Spring launchers deploy electrodes on the surface to measure the horizontal E-fields (4 electrodes on the surface yield 2 orthogonal components), and a fifth on a mast referenced to the others yields the vertical field (Fig. 16). Three-component fluxgate and search coil magnetometers, deployed on a simple (one joint) 1.5-m boom, measure low- and high-frequency magnetic fields, respectively.

Recovery of the horizontal E-field depends only on knowing the sensor locations, and this will be accomplished by imaging the deployed electrometers with the surface cameras. If the sensor location is known to within $\sim 0.1 \mathrm{~m}$, the E-field error is negligible $(<0.5 \%)$. The sensors are designed to survive the full lunar temperature range and are heated by their own power dissipation.

Data rates The EMS has a 50\% duty cycle during the lunar day and a $1 \%$ duty cycle during the night. This short duration during the night still samples frequencies $>10^{-3} \mathrm{~Hz}$ that form the basis of the deep mantle investigations. The EMS operates in two modes: low- and high-rate. Low-rate acquisition from the electrometers and magnetometers at 20 samples/s (sps) is the norm and covers the mantle and core objectives. The high-rate acquisition at $256 \mathrm{sps}$ allows for intracrustal sounding and occurs only for $10 \mathrm{~min} /$ day during daylight and $1 \mathrm{~min} /$ day during the night. With 2 times onboard lossless compression, the 
data rates are $1.1 \mathrm{~Gb} /$ lunation. The EMS has $16 \mathrm{~Gb}$ of NVRAM and can store several months of data, although data would be automatically downloaded to the lander at the beginning of each lunar day and downloaded at $24 \mathrm{~h}$ intervals during the day.

Lander and environmental noise The electrometers and magnetometers are deployed away from the spacecraft at distances that minimize interference and contamination from other spacecraft components. A standard electromagnetic interference/compatibility (EMI/EMC) control plan would be implemented that follows GSFC-STD-7000 ("GEVS").

Mass, and power The full EMS payload allows broadband, vector characterization of both electric and magnetic fields that optimizes the magnetotelluric experiment. The full payload has a mass of $7.2 \mathrm{~kg}$ and uses $3.3 \mathrm{~W}$. Several descope options are possible including eliminating the vertical mast, elimination of the high-frequency search-coil magnetometer, and removal of two electrometers (creating one dipole antenna for RAR). The core science payload includes a fluxgate magnetometer and 4 surface electrodes with a mass of $6.2 \mathrm{~kg}$.

\subsection{Seismometer (SEIS)}

The seismometer [44] is based on the IPGP (France) instruments that are being developed for JAXA's SELENE-2 mission and NASA's GEMS mission. For the full science payload, it is composed of a sensor assembly that encloses 3 long period (LP) oblique seismometers, 1 vertical and 2 horizontal short period (SP) sensors, secondary sensors (temperature, tiltmeters), a leveling device, and acquisition and control electronics. For the core science payload, the two horizontal short-period seismometers are removed. The LP [38] and SP [74] sensors are based on different transducer technologies to reach the best performances in their bandwidth: the LP is an inverted leaf-spring pendulum with a highly sensitive displacement transducer and feedback, while the SP is a diaphragm geophone with a high-efficiency coil-based velocity sensor. The SP geophone had been specially developed for the Moon in the framework of the cancelled JAXA Lunar-A mission.

Deployment and shielding The deployment system is locked during launch, cruise and landing. The seismometer, along with a thermal tent extending $0.5 \mathrm{~m}$ from the outer diameter, is deployed by the robotic arm on the surface $\geq 1 \mathrm{~m}$ from the lander. Once deployed, the sensor assembly is mechanically decoupled from the thermal tent. The seismometer deployable feet penetrate the regolith to ensure sufficient coupling with the ground, which is required for the SP sensor, but not for the LP. Leveling is performed by the seismometer itself. An alternative deployment system for the seismometer would be a simple arm rotating around a vertical axis equipped with a hoist. This would allow a $1 \mathrm{~m}$ deployment from the lander, and would increase the mass by $1.7 \mathrm{~kg}$. 
Data production and performance The measurement objectives dictate continuous data collection. During the day, the instrument records 24-bit data continuously at 20 samples/s for the LP sensor, and 100 sps for the SP sensors. To save power during the night, the LP and SP acquisition frequencies are reduced to 2 and $50 \mathrm{sps}$, respectively. The resolution and noise of SEIS represent major improvements over the Apollo instruments (Fig. 17). In the Apollo bandwidth $(0.1-10 \mathrm{~Hz})$ the LP and SP are 10-20 times better in resolution than the best Apollo output, and digitization noise is below sensor noise. Frequencies outside the Apollo bandwidth are explored for the first time by SP $(10-40 \mathrm{~Hz})$ and by $\mathrm{LP}(<20 \mathrm{mHz})$.

Mass, power, and data budgets In the core science version, the weight of SEIS is $6.2 \mathrm{~kg}$. During daylight, the seismometer power is $2.6 \mathrm{~W}$ and $4.9 \mathrm{Gbits}$ of raw data are generated over 14 Earth days. The night mode allows a reduction of power consumption down to $1.6 \mathrm{~W}$ without a reduction in the data rate. For the full science version, the additional two SP sensors increase the weight to $7.0 \mathrm{~kg}$, though the power consumption remains unchanged. The seismometer records continuously during both lunar day and night, generating $22.4 \mathrm{~Gb}$ of raw data per lunation. Limitations in downlink volume make it impossible to return all data at the highest instrument rates, but much of the data, particularly between discrete events, is of limited value. A quick-look selection strategy based on the vertical SP leads to 6.3 Gbits of data downloaded per lunation and on board compression would reduce this by a factor of about 2 .

Fig. 17 Comparison of the Apollo and SEIS frequency responses. Apollo curves (black) are estimated during non-seismic periods, and SEIS (red and green) are from hardware tests. SEIS is considerably more sensitive and possesses a wider frequency response than Apollo

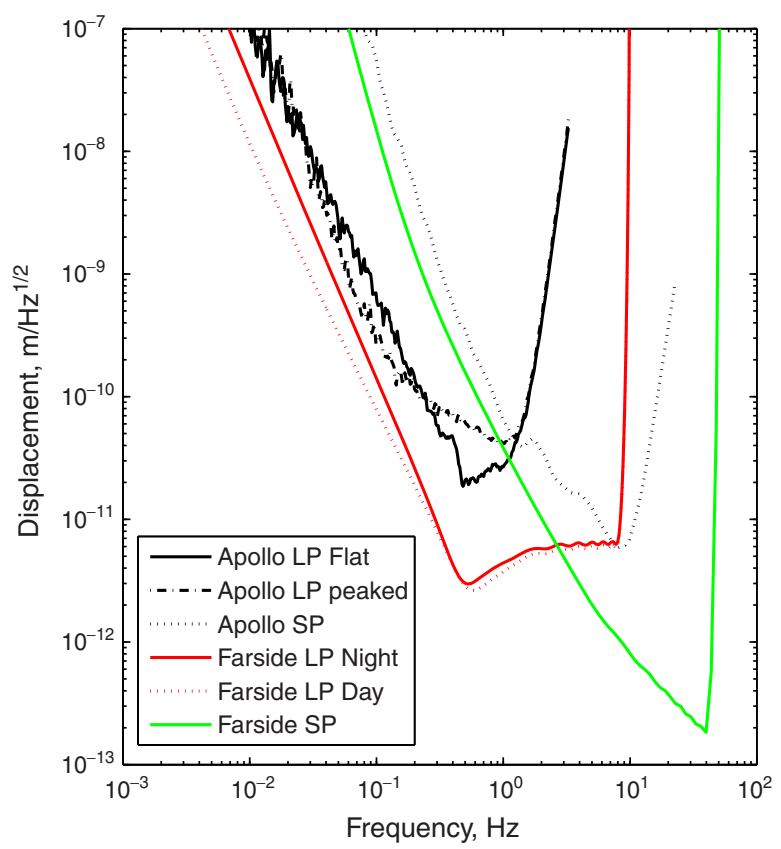




\subsection{Heat flow probe $\left(\mathrm{HP}^{3}\right)$}

The Heat Flow and Physical Properties Package $\left(\mathrm{HP}^{3}\right)$ that is being developed by DLR (Germany) is an experiment that will make measurements to a depth of $3 \mathrm{~m}$ below the surface by means of an electro-mechanical hammering mechanism (Fig. 18). The instrument consists of the five subsystems: the mole that houses the internal electro-mechanical hammering mechanism to provide penetration into the regolith; the payload compartment (PC) that includes acceleration and tilt meter sensors, front-end electronics, soil heaters/sensors and a permittivity probe (PP); an instrumented tether that provides power and data link between the mole and surface and that acts as a carrier for the temperature sensors for the thermal gradient measurement; the support system that provides secure in flight storage of all subsystems; and the back end electronics that are located in the lander. The $\mathrm{HP}^{3}$ sensors include the temperature sensors and heaters that measure the thermal gradient and thermal conductivity using a line-heat source approach; motion and tilt sensors that determine the instrument position in the ground; and electrical sensors that determine relative permittivity of the regolith.

Deployment and performance The $\mathrm{HP}^{3}$ is to placed on the ground by a robotic arm more than 1 meter away from the lander. If a robotic arm is not provided, a dedicated unfoldable boom could also accomplish deployment. Such a system weights approximately $2 \mathrm{~kg}$, has a single degree of freedom, and is capable of a $3 \mathrm{~m}$ deployment distance.

Fig. 18 Tractor mole (left; $2.5 \mathrm{~cm} \times 25 \mathrm{~cm}$ ) and support system (right)

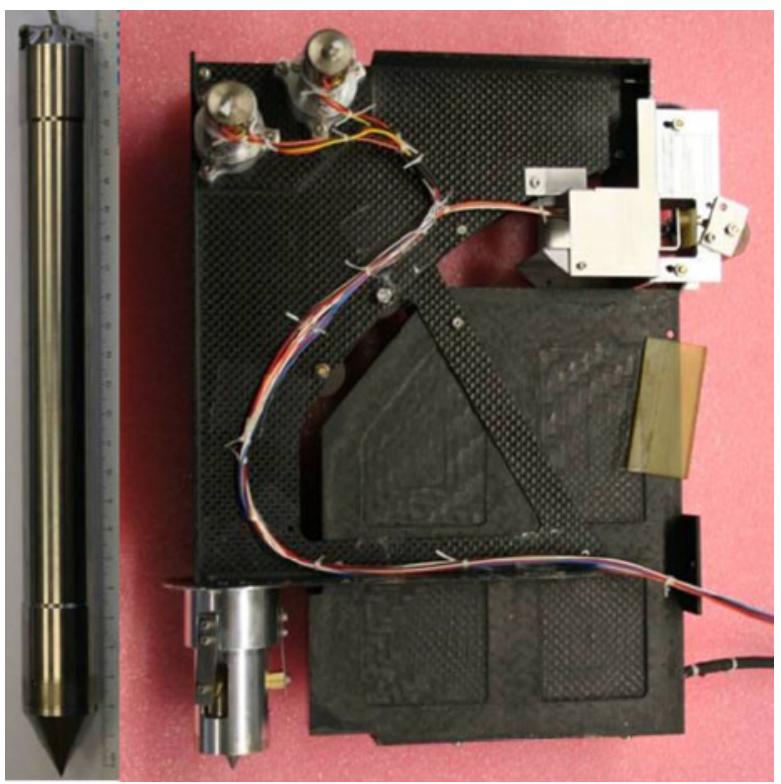


During penetration, which takes up to 6 lunations because of intervening thermal conductivity and permittivity measurements, the mole displaces any subsurface rocks that are less than 2 times the mole diameter or deflects from larger rocks. If penetration is stopped before reaching the target depth of $3 \mathrm{~m}$, heat flow measurements can still be achieved, but with reduced accuracy. The minimum penetration depth to achieve the science goal is $1.5 \mathrm{~m}$, provided that measurements are extended over at least a full year to characterize annual temperature fluctuations. In this case, the heat flow uncertainty would increase from 10 to $15 \%$.

There are two phases to instrument operations. During penetration, soil intrusion is achieved by electro-mechanical hammering. The net hammering time is expected to be $\sim 24 \mathrm{~h}$ to reach the final depth of $3 \mathrm{~m}$, but hammering is interrupted every $30 \mathrm{~cm}$ to conduct thermal conductivity and permittivity measurements. A period of up to 10 Earth days after each hammering episode is needed to reach a sufficiently equilibrated thermal state before each measurement starts, and heating then takes $168 \mathrm{~h}$. Permittivity measurements can be conducted within a few minutes. The monitoring phase begins when terminal depth has been reached, and consists of hourly passive temperature readings.

Thermal control No survival power is required when the instrument is switched off. However, electrical heaters in the mole and in the front-end electronics are required to thermally condition the instrument prior to any operations. The back-end electronics are accommodated in the lander, and the components of the system that require thermal control are designed for the surface environment conditions.

Mass, power, and data budgets The weight is estimated at $1.5 \mathrm{~kg}$ for a complete mole system and $2.8 \mathrm{~kg}$ for a dual-mole system. Maximum power consumption occurs during hammering and is $10.2 \mathrm{~W}$, with a minimum during monitoring at $2.6 \mathrm{~W}$. During night, only temperature monitoring every hour is performed and the power consumption is negligible. Data rates are extremely low, at $0.1 \mathrm{Mb} / \mathrm{hr}$ during hammering, $8 \mathrm{~kb} / \mathrm{hr}$ during thermal conductivity measurements, and $8 \mathrm{~kb} / \mathrm{hr}$ during monitoring.

\subsection{Context camera (CAM)}

Critical surface operations include the deployment of the seismometer and heat flow probe onto the surface. Cameras on each lander are required to (1) establish the geological context of the geophysical measurements, (2) enable and support instrument deployment by providing $360^{\circ}$ panoramas and 3D stereo images of the lander's surroundings, and (3) select instrument deployment sites and eventually deploy the instruments. Furthermore, image data will allow a variety of surface monitoring studies. One camera will be accommodated on the robotic arm, and another on the deck of the lander to monitor the arm. By moving the robotic arm, a $360^{\circ}$ panorama will be provided 
with a 3D stereo reconstruction. Comparison with high-resolution imagery from orbit will allow refinement of the exact position and orientation of the landers.

Instrument design The instrument suite consists of two wide-angle cameras that are being developed by DLR (Germany). Each camera head is accommodated in its own mechanical housing, and the two cameras share a common electronics box and processing unit. Each detector head contains an optical lens assembly and a CIS detector, and both cameras use identical $1024 \times 1024$ pixel detectors. A focal length of $6.7 \mathrm{~mm}$ is used (equisolid fisheye) providing a field of view of $140^{\circ} \times 140^{\circ}$. At a distance of $0.8 \mathrm{~m}$, the angular resolution at the center of the image is $\sim 2.24 \mathrm{mrad} / \mathrm{pixel}$, and the spatial sampling at the center of the image is about $1.8 \mathrm{~mm} / \mathrm{px}$. If a stereo baseline of $0.5 \mathrm{~m}$ is used, the stereo angles would be $7.1^{\circ}$ at $4 \mathrm{~m}$ and $31.1^{\circ}$ at $0.8 \mathrm{~m}$, and the stereo reconstruction accuracy would be $0.07 \mathrm{~m}$ at $4 \mathrm{~m}$ and $5 \mathrm{~mm}$ at $0.8 \mathrm{~m}$.

Mass, volume, and data rates The envelope of the proposed camera (excluding control electronics) is $6 \mathrm{~cm} \times 6 \mathrm{~cm} \times 6 \mathrm{~cm}$ and the total average power is $1.5 \mathrm{~W}$. The mass is $0.2 \mathrm{~kg}$ per camera head and approximately $0.6 \mathrm{~kg}$ for a common camera main electronics and data processing unit. Stereo images of the landing site and images taken during instrument deployment will account for less than 1 Gbits, which represents less than one-tenth of the data downloaded from the lander each lunation. Images for science purposes will account for $\sim 1$ Gbit/lun.

\subsection{Neutron Gamma-ray Spectrometer (NGS)}

Neutron and gamma-ray spectroscopy is a standard technique for measuring planetary surface compositions. Neutron and gamma-ray spectrometers have flown previously on missions to the Moon, Mars, Mercury, and asteroids, and a miniaturized version of these orbital instruments has been proposed for in situ measurements on planetary surfaces [14]. The measurement technique uses neutrons and gamma-rays that are produced by galactic cosmic rays, and the returned data consists of energy pulse height spectra integrated over a commandable-length time cadence. The instrument includes modules that contain ${ }^{3} \mathrm{He}$ neutron sensors and gamma-ray scintillator sensors, and the acquisition and processing electronics would be housed in a dedicated module in the lander.

Sensor placement, thermal control and performance The NGS has separable sensor and data modules so that the sensors can be located in optimum locations near the surface. The ideal placement of the sensor modules would allow a $2 \pi$ viewing of the lunar surface, such as provided by the side or bottom of the lander. Placement of the sensor modules on a short boom, or deployment to the surface by a robotic arm, would provide a reduction in background from neutrons and gamma-rays generated by the interactions of cosmic rays 
with lander materials. The NGS sensors and electronics operate within a temperature range of $\pm 40^{\circ} \mathrm{C}$ and need no special thermal accommodation beyond what would be provided for other instruments. When turned off, the sensors would survive the night on the lunar surface. No special pointing is required because the sensors view all space.

Mass, power, and data budgets The mass of the entire NGS would be no more than $2.5 \mathrm{~kg}$. The neutron spectrometer itself could be built for $\sim 500 \mathrm{~g}$ [14], and the primary mass driver is the gamma-ray scintillator. The overall power utilized by the neutron gamma-ray spectrometer is less than $4 \mathrm{~W}$, and since nighttime measurements are not required, the instrument would be turned off during the night to conserve power. The total data volume per gamma-ray and neutron spectra accumulation would be less than 4 Kbytes, and as fast time measurements are not required, the total data rate could be made quite low. If data were accumulated over one-hour intervals, the total data rate would be close to 1 byte per second, for about 1 Mbits per lunation. Even for a scenario where data are collected with a cadence of about a minute, the data rate would be no more than 0.1 Gbits per lunation.

\subsection{Impact monitoring camera}

The impact-monitoring camera is based on a modified version of the Smart Panoramic Sensor Head (SPOSH) that is being developed by DLR (Germany). SPOSH is an assembly of detectors to observe meteoroid impacts and possible other luminous night time phenomena on the farside of Moon [50], and will allow detection of any meteoroid impact with a mass larger than a few grams. The camera consists of two main parts: SPOSH-VIS for observations in the visible $(400-800 \mathrm{~nm})$, and SPOSH-IR for observations in the near infrared (1000-2500 nm). Each of these contains separate camera heads that consist of an optical telescope with an approximately $0.5 \mathrm{~m}$ focal length and a detector unit. Both SPOSH units are enclosed in a common housing, and a common digital processing unit (DPU) on flexible printed circuit boards is folded around the camera heads.

Deployment and performance Operations would start after the positioning of the orbiter in the lunar L2 halo orbit. Images of star fields would be used for geometric and radiometric calibration of the two camera heads. The SPOSH DPU uses powerful event-detection software, and in typical operations the DPU would reduce the data stream dramatically by transmitting only those portions of images that contain events. During the commissioning phase, full image frames would be stored and transmitted to Earth for evaluation. The instrument must be pointed so that the Sun does not enter the instrument's narrow field of view.

Thermal control The operating temperature of the VIS and IR detectors is $-20^{\circ}$ and $-80^{\circ} \mathrm{C}$, respectively. The preferred cooling mechanism is radiative 
cooling by radiators to free space, though cooling by Peltier elements is a power-consuming alternative. The operating temperatures of other parts of the instrument are about $10^{\circ} \mathrm{C}$.

Mass, power, and data budgets The mass of the instrument is estimated to be $10 \mathrm{~kg}$, and the required power is lower than $8 \mathrm{~W}$. An additional $10 \mathrm{~W}$ for each sensor would be required in the case of an active cooling system. The overall instrument dimensions are approximately $13 \mathrm{~cm} \times 30 \mathrm{~cm} \times 25 \mathrm{~cm}$. For an estimated 1000 observed events per year 10-20 Mbytes of event data are expected, or about $25-50$ Kbytes per day. However, the data rate may be variable, and during meteoroid showers, data rates may increase 20 -fold.

Data quality To assure high data quality, low detector temperatures have to be maintained. To ensure event localization with pixel precision, the pointing stabilization is required to be better than $\sim 8 \mathrm{arcsec} / \mathrm{s}$, and the pointing knowledge better than $\sim 4$ arcsec. Impact events will be localized in time to better than $0.3 \mathrm{~s}$, and the spatial resolution will be determined by the chosen focal length.

\subsection{Magnetometer (MAG)}

The magnetometers on the relay satellite are based on those being developed by the Technical University of Denmark. They consist of two sensor heads and a common electronics box connected to the sensors. One of the key parameters of the magnetometer is the zero-level accuracy of the measured vector field.

Deployment and thermal control As magnetic cleanliness requirements are not being considered for the relay satellite, the sensor heads must be mounted on a 3-4 $\mathrm{m}$ long boom in order to minimize magnetic noise from the spacecraft. By placing one sensor head at the end of the boom and the other closer to the spacecraft, any remaining magnetic noise will be estimated. In order to timestamp the measurements with sufficient accuracy, a synchronization signal needs to be provided to the instrument. The instrument electronics operate in the temperature range $\pm 50^{\circ} \mathrm{C}$ and can survive in the range $\pm 60^{\circ} \mathrm{C}$.

Performance The magnetometer dynamic range is $\pm 65536 \mathrm{nT}$ to $0.0625 \mathrm{nT}$ (21 bits), and its linearity is $0.0001 \%$ of the full scale. The intrinsic sensor noise is as low as 15 pT RMS in the band $0.05-10 \mathrm{~Hz}$, and the intrinsic electronics noise is $35 \mathrm{pT}$ RMS in the same band. The thermal and long-term zero stability are less than $\pm 0.2 \mathrm{nT}$, and the thermal offsets are $\sim 0 \mathrm{nT} /{ }^{\circ} \mathrm{C}$ for the sensor and $\sim 0.05 \mathrm{nT} /{ }^{\circ} \mathrm{C}$ for the electronics.

Mass, power, and data budgets The mass of each sensor is $150 \mathrm{~g}$, the mass of the electronics is $800 \mathrm{~g}$ and the harness is $80 \mathrm{~g} / \mathrm{m}$. The total power consumption is $2.5 \mathrm{~W}$. The sampling frequency is $50 \mathrm{~Hz}$ and the data rate corresponds to $\sim 200$ bytes/second. 


\section{System requirements and spacecraft issues}

The mission requirements derive from the three main mission phases: Launch and early operations (LEOP) and transfer, lunar arrival, and descent.

LEOP and transfer This phase starts with a launch from Kourou to GTO of the two identical spacecraft and orbiter, mounted one on top of the other. The spacecraft separation relies on the same strategy as for Cluster-2, where the upper composite (the upper lander and relay satellite) is separated by commands via an umbilical that is wired through the lower vehicle. A few minutes later, the lower vehicle is released. From this point onwards, the two spacecraft fly separately during their transfer to the lunar L2 halo orbit. Communications are achieved through spacecraft omni-directional X-band low gain antennas to $15-\mathrm{m}$ terrestrial ground stations.

Lunar arrival The lower lander and composite arrive near LL2, and are injected into a 14-day halo orbit. The relay satellite is then separated from the lander, and the first in-orbit tests are performed on the relay satellite, as it is required to be operational for the landing of the lunar probes. The relay satellite is to operate for the nominal lifetime of 4 years, and carries UHF/X band relay capabilities and a small science payload.

Descent After 15 days to 1 month, the first probe is inserted into a ballistic trajectory down to the Moon. At about $20 \mathrm{~km}$ altitude, the main braking maneuver is initiated. The attitude of the spacecraft is slewed into its landing attitude and landing is autonomously performed using a Doppler radar altimeter. The whole process is performed under ground control in real-time. After one month ( 15 days of first lander installation, plus 15 days of lunar night) the descent of the second lander begins, following the same procedure.

The nominal operational life of the lander is 4 years. Time synchronization of the landers, which is required by the science payload, will be performed through the lander-satellite link. The initial localization of the landers on the surface will rely on differential Doppler measurements and lander star-tracker attitude and inertial measurements. Comparing images from the surface with existing high-resolution Lunar Reconnaissance Orbiter images and topography will enable a more precise localization. The high precision required by the radio astronomy interferometric measurements will be eventually reached using known radio-sources.

\subsection{Lander spacecraft requirements}

\subsubsection{Lander outline}

Given the stringent mass constraints on the lander, a design relying on the heritage of previous lunar lander studies (Astrium Satellites "MoonTwins") is chosen (Fig. 19). With many simplifications at GNC and propulsion level, 


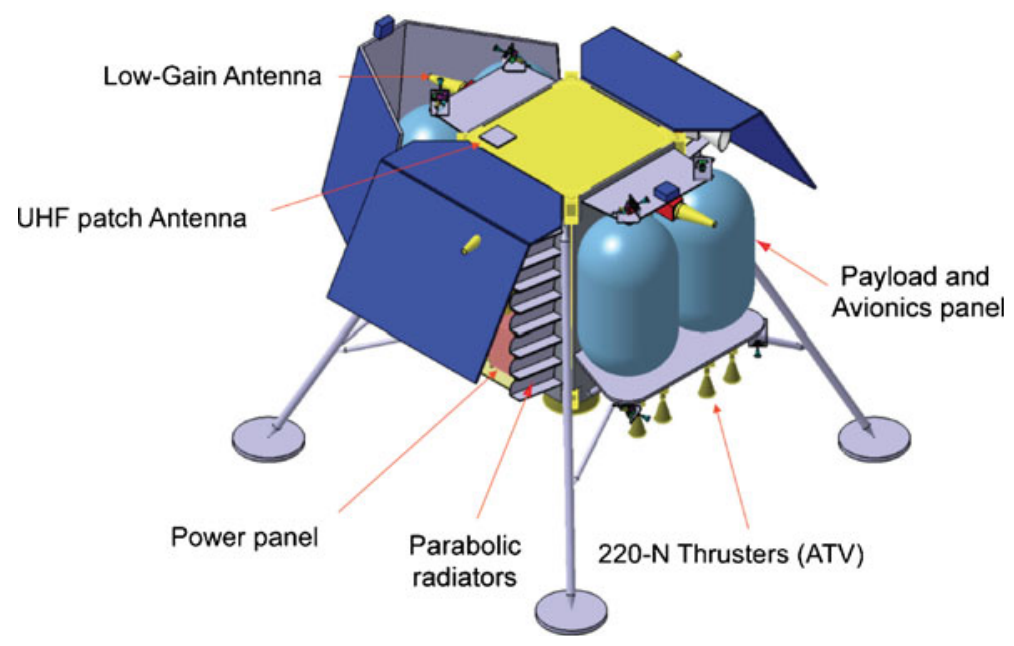

Fig. 19 Lander preliminary accommodation. After landing, an arm (not shown) will deploy the seismometer and heat flow probe on the ground (Image courtesy of Astrium Satellites)

the proposed concept is still significantly heavier than the JPL "Lunette" and "Lunar Geophysical Network" landers [60] that have a similar payload mass.

The power system relies on solar power provided by fixed solar panels and enough batteries to enable a minimum set of instruments to operate during the night at reduced levels. An automated wake-up system is implemented in the power system to restore avionics to nominal operations at sunrise, and to allow a minimum operation level during the night. The avionics architecture is based on a dual structure: the command and data management unit (CDMU) contains the processor module and memory, and the electrical interface unit (EIU) manages the interface and power lines with the attitude and orbit control system (AOCS), the propulsion units, and the heater lines. The architecture is based on an RS422 interface bus, and has the ability to be switched on and off by a timer to allow the minimization of energy spent during the night.

\subsubsection{Attitude and orbit control}

The GNC system makes use of an efficient set of sensors that are able to handle all of the mission's phases, including a star tracker, an inertial measurement unit (gyrometers-accelerometers), a Doppler-radar altimeter, Sun acquisition sensors, and MEMS gyroscopes as spare sensors during safe mode for the spacecraft. An alternate option would be to use a camera for the landing. The actuators are all propulsive, based on a high thrust system for landing (one 500 $\mathrm{N}$ main engine and four ATV $250 \mathrm{~N}$ thrusters) and a low thrust reaction control system (RCS) for fine attitude and orbit control with eight $10-\mathrm{N}$ thrusters. An alternate design, using solid-rocket motors, such as ATK STAR 30BP, could also be considered for the final descent braking. 


\subsubsection{Power}

The power subsystem is based on a classical design (PCDU, unregulated $28 \mathrm{~V}$ ). The available power provided by the solar arrays is about $200 \mathrm{~W}$ during the day, and the available power during the night is about $4 \mathrm{~W}$. Night operations for the payload will be mostly in a low power mode and with a duty cycle that is compatible with the battery resources. A low energy consumption mode is used for payloads that remain on during the night, with all possible bus equipment switched off. Only a timer for the lander wakeup and the power conditioning and distribution unit (PCDU) for the payload power supply remain on. Small periodic wake-ups (with a negligible impact on energy budget) are used to store data in the CDMU and perform basic communications, such as time synchronization of the landers with the orbiter. Data will be stored at instrument level during the night and uploaded during the day.

\subsubsection{Data handling and telemetry}

Telecommunications with Earth are handled differently depending on the mission phase. During LEOP and ballistic transfer, $\mathrm{X}$ band is preferred, and an omni-directional coverage is provided by means of two low gain antennas. For the science mission phase, a UHF antenna provides the low-rate satellite to lander link, which limits the data upload to $128-256 \mathrm{kbps}$, depending on the final relay orbit chosen. Supposing an uplink during the lunar day only, it allows for between 150 to 300 Gbits of data transfer per lunation. The UHF link is also used to allow time synchronization of the two landers through the orbiter. The high data rate X-band link could also be used to allow for high rate uploads.

\subsubsection{Thermal concept}

Surviving the lunar night, while keeping enough resources for minimum payload operation and keeping the avionics warm to operate during 14 terrestrial days, is one of the biggest technical challenges of this mission. Two concepts have been considered that have a considerable impact on the lander spacecraft sizing. The baseline concept makes use of radioisotope heating units (RHUs) to keep the critical parts of the spacecraft (batteries and sensor electronics) warm and uses a second surface mirror (SSM) shadowed from the Sun to dissipate the excess heat during the day. While it is clear that Pu-238 shortages make this option difficult, ESA has started to consider alternative radionuclides for RHUs, such as Americium-241. This isotope has a longer half-life than plutonium-238, but produces less heat and electricity, making the technical solution less mass-efficient.

An alternate design that does not make use of RHUs has been also studied but it has severe consequences on spacecraft design. Keeping the critical 
avionics warm during the night requires a very good insulation of the warm electronics box, which in turn leads to potential overheating during the day. Our proposed technical solution makes use of thermal switches (which are lighter than variable capacitance heat pipes) and parabolic shaped radiators to allow sufficient heat rejection during the day (Fig. 20).

\subsubsection{Lander spacecraft mass and power budgets}

A preliminary estimate of the lander mass budget confirms the consistency of the spacecraft budgets with the system mass allocation. The lander mass with the full science payload is far below the mass allocations for the Ariane 5 shared launch option (Table 4). The lander mass with the core science payload is marginally compatible with a Soyuz launch: the lander mass maturity margin is in this case $16 \%$ instead of the required $20 \%$ maturity margin. This is however compatible with the proposed use of already available, off-the-shelf equipment. Cruise and daytime power budgets are about 180 and $150 \mathrm{~W}$ with

Fig. 20 Thermal concept using thermal switches and radiators, inspired from Apollo design. This radiator configuration allows for very efficient heat rejection in the day. The solid angle under which the radiator sees the ground is reduced, and heat is rejected in a direction parallel to the ground. During the night, the thermal switch is closed to preserve heat in the electronics enclosure

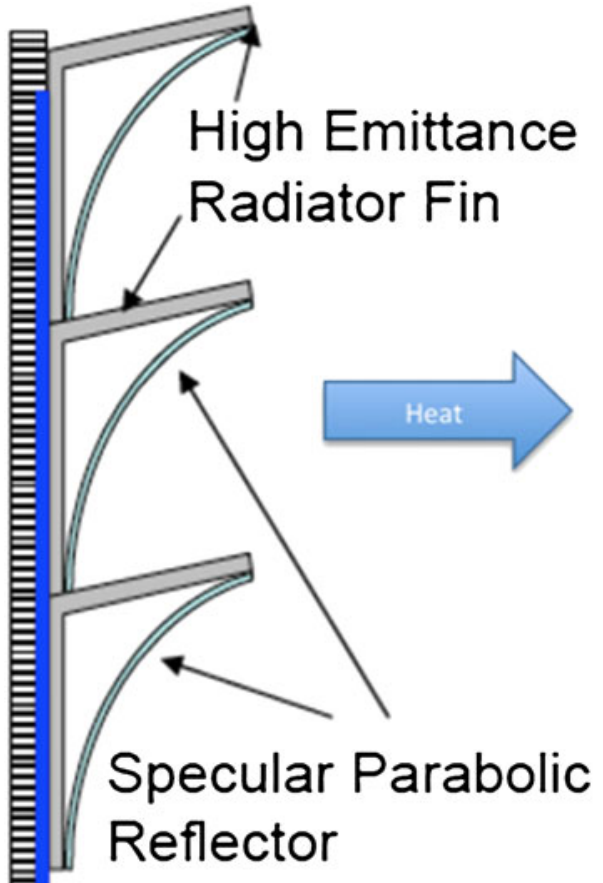

Thermal switch off during night

To Warm Electronic Enclosure 
Table 4 Preliminary lander mass budget
The mass of the lander with the full science payload is well below the mass allocated for an Ariane V launch. The mass of the lander with the core science payload is marginally consistent with a Soyuz launch, with a $16 \%$ maturity margin

\begin{tabular}{lcc}
\hline Lander subsystem & $\begin{array}{c}\text { Current best } \\
\text { estimate }\end{array}$ & $\begin{array}{c}\text { With 20\% } \\
\text { margin }\end{array}$ \\
\hline Structure & 56 & 67 \\
Harness & 18 & 21 \\
Landing gear & 40 & 48 \\
Propulsion & 100 & 120 \\
EPS system & 33 & 40 \\
C\&DH & 11 & 13 \\
AOCS \& GNC & 12 & 14 \\
TTC & 15 & 18 \\
Thermal HW & 18 & 22 \\
Full Science Payload & 28 & 33 \\
Core Science Payload & 22 & 27 \\
Total Lander (Core Science) & $\mathbf{3 2 5}$ & $\mathbf{3 9 0}$ \\
Total Lander (Full Science) & $\mathbf{3 3 0}$ & $\mathbf{3 9 6}$ \\
Lander Allocation (Soyuz) & & 380 \\
Lander Allocation (Ariane V) & & 475 \\
\hline
\end{tabular}

and without communications, respectively. During the night, the preliminary design allows $4 \mathrm{~W}$ for the overall payload and bus consumption.

\subsubsection{Critical issues}

Redundancy As the science objectives require a long life on the lunar surface, the overall redundancy philosophy is linked to the choice of a good reliability during the mission. We have chosen to have redundancy for all units operational after the landed phase, but not for the transfer phase (CDMU, EIU, Transponders, and PCDU). Thrusters are also not redundant (only 4 of the 8 in the "MoonTwins" design are required). In addition, one can consider that the use of two identical landers provides a de facto redundancy to the mission, enabling the threshold science to be reached even with the loss of a lander.

Lander cost One of the most critical aspects is the need for the development of a light lunar lander, as required for the Soyuz-Fregat launch option. Our approach is made possible in that it relies on already proven technologies at the subsystem level. Only high TRL equipment (such as Eurostar or ATV boosters) is considered and redundancy (with respect to the "MoonTwins" study) has been reduced to a minimum: no new developments such as hazard avoidance or high-precision landing systems are required by the proposed concept. Development synergies with the ESA exploration program lunar lander [57] are evident, and cooperation with another partner concerning the relay satellite could also relax the cost constraints.

Electromagnetic cleanliness Electromagnetic and radio astronomy measurements require that a special attention be paid to the overall electromagnetic 
cleanliness of the landers. Standard techniques are to be used, as implemented on missions such as Ulysses, Stereo, BepiColombo. DC magnetic fields, however, are not among the primary science objectives on the landers.

\subsection{Relay satellite requirements}

\subsubsection{Satellite outline}

The requirements of the relay satellite are to accommodate a small scientific payload (an impact flash camera and magnetometer) while providing a telecommunications relay for the two landers. A tailored Myriad-class (CNES mini-satellite) or mini-geo bus design is considered to accommodate the scientific payload (Fig. 21). Even if it is designed for a shorter life duration (1 year), this satellite class (Demeter and Parasol missions) has already proven reliability compatible with our requirements. The control of the satellite attitude allows satellite orientation on 3 axes. In the nominal mission, the relay satellite is oriented along a North-South line, with the relay antenna on its "East" side. The satellite remains pointed towards the night side of the Moon for impact monitoring.

To allow minimal changes to on-board software with respect to existing designs, the satellite follows a tabulated attitude as a function of the orbit (pseudo-)period, and the solar panels rotate with the Earth-Moon synodic period. To enable the telecom relay capability (Fig. 22), two options are considered: either an X-band beam forming network (BFN) would keep a synthetic tracking of Earth, or Earth pointing would occur when impact flashes could not be observed (such as when the farside disk is fully illuminated by the Sun). Satellite pointing precision is about $5 \times 10^{-3}$ arc degrees, which is sufficient for the impact flash detection payload. In the nominal mode, the satellite uses a stellar sensor and four reaction wheels, with Sun sensors being

Fig. 21 The relay satellite is based on a micro-satellite platform. The satellite includes the impact monitoring camera on the front side, and a deployable high gain antenna on the side. The magnetometer (not shown) is to be deployed on a boom

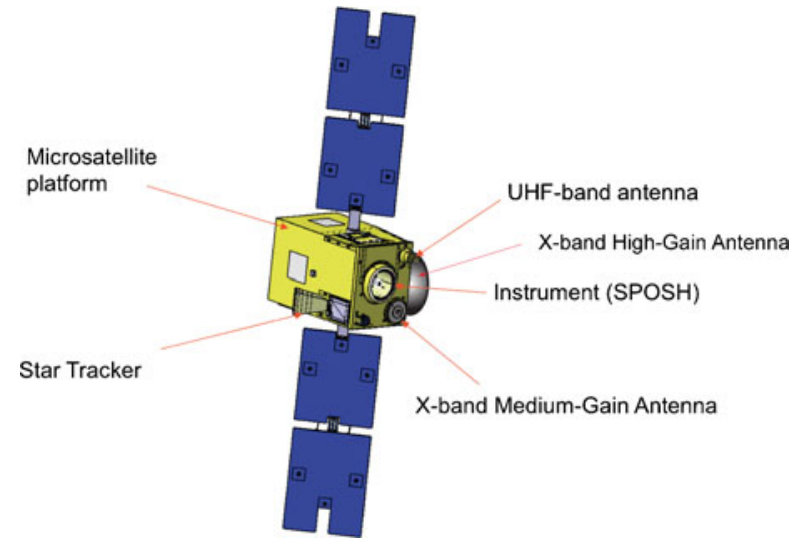




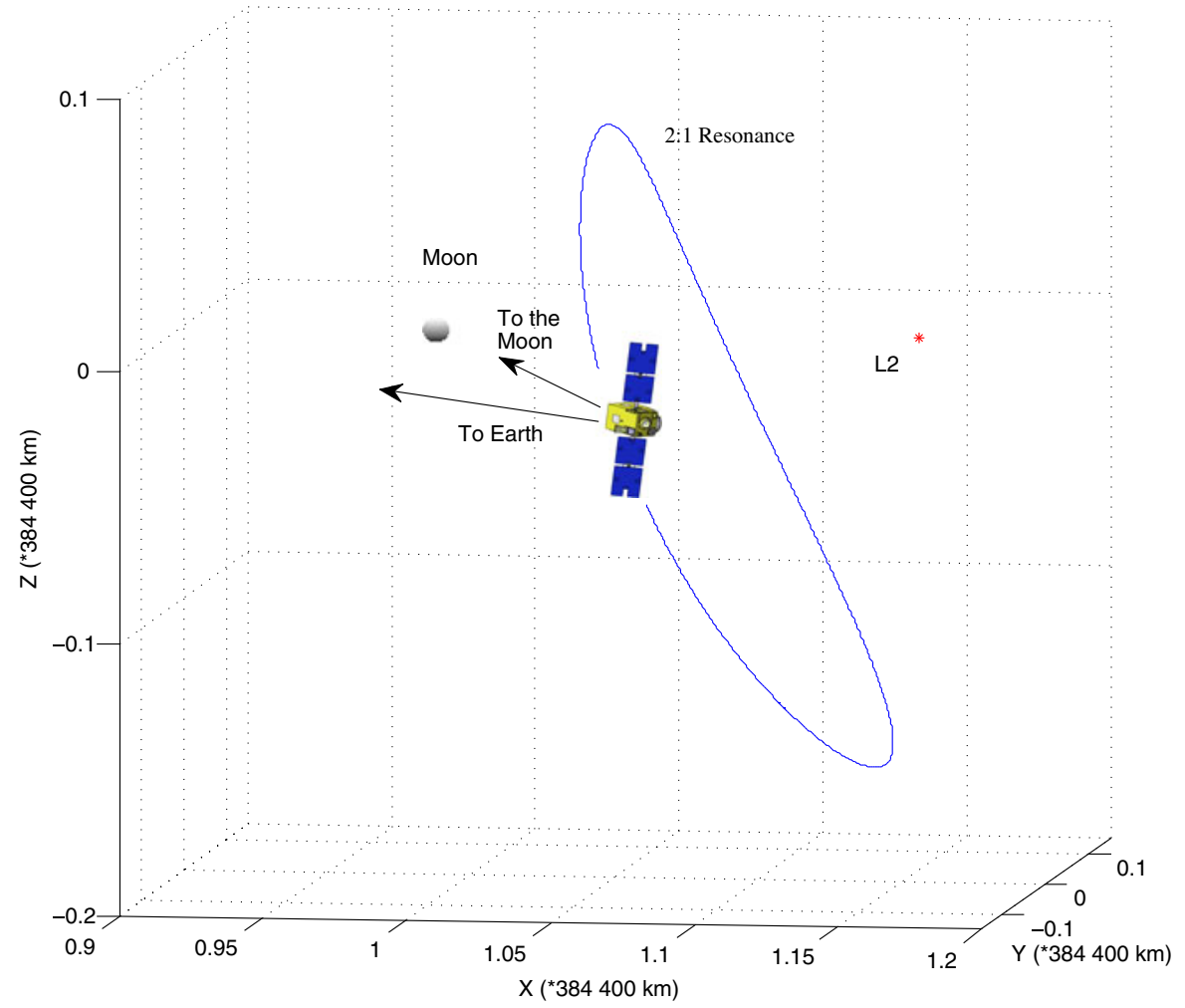

Fig. 22 Relay satellite pointing geometry. The mission requires two different pointings: one to the Moon for the impact flash monitoring experiment and to provide a relay-link to the landers, and another to track the Earth stations. The baseline is to share time between these two pointings

provided for safe mode. Momentum wheel desaturation is performed with cold gas thrusters.

\subsubsection{Electrical power subsystem}

The electrical power subsystem (EPS) derives from the Myriad power generator, but uses 4-hinged panels (folded during launch) instead of 2. The overall surface area of the solar panels is approximately $1.6 \mathrm{~m}^{2}$, which allows $360 \mathrm{~W}$ of power as a result of the use of highly efficient triple junction AsGa cells. Solar panels rotate to follow the Sun using a solar array drive mechanism. The power supply uses a solar generator constituted of two hinged panels folded against the platform during launch that can rotate around an axis through a drive mechanism. The system also includes a $\mathrm{Li}$ ion battery and an electronics box supplying a regulated power to all spacecraft equipment. 


\subsubsection{Data handling and telemetry}

The overall architecture of the satellite is centralized. A serial data bus using a star architecture ensures communications with all equipment. The Myriad avionics are largely based on commercial off the shelf components (COTS) for which the reliability has been demonstrated (operation for several cumulated years in orbit). It decodes and executes the commands from the ground, formats housekeeping and science telemetry, manages the on-board data distribution, and does all real-time computations onboard. The flight software runs on the CDMU, which contains a T805 processor with 256 Mbytes of flash memory and 1 Gbits of RAM. A solid-state mass memory is used to store housekeeping and science data telemetry until its transmission to the ground. Due to the severe environment, redundancy of critical systems could be considered. A UHF local link is used for communication between the probes and relay satellite (following the Proximity-1 Space Link Protocol) with an uplink capability of about 128 to $256 \mathrm{kbps}$. An X/S band link is used for direct communication of the relay satellite to Earth.

\subsubsection{Relay satellite mass and power budgets}

The mass of the relay satellite is about $150 \mathrm{~kg}$, including its $50 \mathrm{~kg}$ payload (this includes the specific TT\&C subsystem of about $40 \mathrm{~kg}$ ). If the satellite were to accommodate the propulsion for the lunar transfer (with a $500 \mathrm{~N}$ ATV bipropellant motor), the total mass of the relay satellite would rise to $250 \mathrm{~kg}$. The orbiter power budget is about $60 \mathrm{~W}$.

\subsection{Mission operations concept}

Ground system functions will be performed by ESA through the launch, cruise, landing and instrument deployment phases of the mission. Routine surface science operations will be performed by ESA for the first 2 years after landing, transitioning to a nationally owned control center for the final 2 years of operations. The contributed national facilities could be available starting at the beginning of routine science operations, so that they could be used as a backup to the ESA sites. The Farside Explorer ground segment will consist of a command and data acquisition element composed of ground stations from the ESOC network, and a mission operation and control element located at ESOC facilities in Darmstadt. The science facilities will be located at the instrument PI's institutions.

Farside Explorer will use primarily telecommunication services from the ESA network. In case of dire need, a request would be made to use the DSN for telecommunications with the two spacecraft, using the 34-m beam waveguide sub-network. The Farside Explorer spacecraft will use standard CCSDS formats and protocols to ensure international interoperability. 
The mission is designed such that except for initial acquisition after launch, simultaneous multiple ESA antennas for mission critical events will not be required for both spacecraft at the same time. The mission will use X-band for all ground communications. After launch and early operations, the tracking for each spacecraft would be dominated by the needs of cruise navigation, which is especially important for the three weeks before landing. Surface deployment is scheduled to be as long as two weeks, corresponding to the maximum length of daylight after landing.

Shortly after launch, the two spacecraft (lander plus composite) separate from the launch vehicle and follow slightly different trajectories to LL2. Each of the lunar probes will then be targeted to landings timed about one lunar month apart. Command sequences for each of the spacecraft will be designed, written, and uplinked on a non-interfering basis so that a sole navigation event (such as a trajectory correction maneuver) will occur at a given time. The landing operational sequence will be run autonomously onboard the spacecraft, and monitored by the operations team. Telecommunications during this phase of operations will be based on the amount of tracking needed for navigation.

The Farside Explorer mission will continuously collect science data over the full lunar day/night cycle. During daylight operations, the lander will operate in its normal mode, continuously collecting engineering data and science data from the instruments. These data will be stored onboard the lander and downlinked continuously to the orbiter. Data taken during the lunar night would be stored in the instrument memory for daytime transfer to the CDMU and subsequent downlinking. Short wake up sequences will occur on a daily basis to allow health check and time synchronization.

\subsection{Current heritage and TRL}

Payload technology Most of the proposed payload has already been developed in the framework of the Cassini, ExoMars, SELENE-2, and GEMS missions and has therefore a TRL level over 5.

Lander technology In order to keep the lander cost low, the platform will use primarily off-the-shelf equipment. The propulsion system has been built around ATV heritage in order to save technological development costs and associated risk, and the avionics require no specific developments. The overall TRL of all baseline subsystems is very high, ranging from 8 to 10 . The only exception is the use of RHUs for the thermal design, which requires additional developments (an ESA TRP is already in progress). If the use of RHUs is not possible, the thermal design will rely on a warm box enclosing the whole electronics, and on the use of thermal switches to handle the hot case. (Thermal switches are currently under qualification in Europe, but could be provided by US partners, under ITAR regulations). 
Relay orbiter technology The relay satellite would be derived from the Myriad bus and would use only standard, often off-the-shelf, technologies.

\section{Summary and conclusions}

Farside Explorer is an innovative medium-size mission to the farside of the Moon that has been developed in the framework of ESA's Cosmic Vision program. The scientific objectives of this mission are designed explicitly to exploit three unique properties of the scientific platform offered by the farside of the Moon.

- The farside of the Moon is shielded from terrestrial radio-frequency interference, and during the lunar night it is also shielded from solar radio emissions.

- The farside of the Moon records the primary differentiation and evolution of this celestial object, and hosts the largest impact basin in the Solar System.

- The farside of the Moon can be monitored continuously from the EarthMoon L2 Lagrange point, where there is a complete lack of reflected solar illumination from the Earth.

With two lunar landers and an instrumented relay satellite, Farside Explorer will exploit these properties.

- Farside Explorer would make the first radio-astronomy measurements from the most radio-quiet region of near-Earth space.

- Farside Explorer would determine the internal structure and thermal evolution of the Moon, from crust to core.

- Farside Explorer would quantify impact hazards in near-Earth space by the measurement of flashes generated by impact events.

One lander would explore the largest and oldest recognized impact basin in the Solar System - the South Pole-Aitken basin — and the other would investigate the primordial highlands crust. Radio astronomy, geophysical, and geochemical instruments would be deployed on the surface, and the relay satellite in a halo orbit about the lunar L2 Lagrange point would continuously monitor the surface for impact events. The ambitious science objectives are enabled by an innovative approach. The flight system can be launched to a geosynchronous transfer orbit by either a Soyuz-Fregat or a shared commercial Ariane 5, and a weak boundary transfer trajectory along the Earth-Sun L1 gravity manifold is used to minimize the $\Delta \mathrm{V}$ of insertion into a lunar $\mathrm{L} 2$ halo orbit.

Acknowledgements The authors would like to thank Astrium Satellites and Thales Alenia Space who contributed to the preparation of this paper. Claude Jaupart, George Smoot, Sean Solomon, and Stuart Ross Taylor are thanked for their support of this mission proposal. For a complete listing of the supporting individuals, visit the web site http://farside.spacecampus-paris.eu/. 
Open Access This article is distributed under the terms of the Creative Commons Attribution Noncommercial License which permits any noncommercial use, distribution, and reproduction in any medium, provided the original author(s) and source are credited.

\section{References}

1. Alessi, E.M., Gomez, G., Masdemont, J.J.: Two-manoeuvres transfers between LEOs and Lissajous orbits in the Earth-Moon system. Adv. Space Res. 45, 1276-1291 (2010)

2. Alexander, J.K., Kaiser, M.L., Novaco, J.C., Grena F.R., Weber R.R.: Scientific instrumentation of the Radio-Astronomy-Explorer-2 satellite. Astron. Astrophys. 40, 365-371 (1975)

3. Bellot Rubio, L.R., Ortiz, J.L., Sada, P.V.: Luminous efficiency in hypervelocity impacts from the 1999 lunar Leonids. Astrophys. J. 542, L65-L68 (2000)

4. Bely, P.Y., Laurance, R.J., Volonte, S., Ambrosini, R.R., Ardenne, A., Barrow, C.H., Bougeret, J.L., Marcaide, J.M., Woan, G.: Very Low Frequency Array on the Lunar Far Side. ESA report SCI(97)2, European Space Agency (1997)

5. Bignami, G., Cargill, P., Schutz, B., Turon, C.: Cosmic Vision. BR-247, ESA, Noordwijk (2005)

6. Boischot, A., Rosolen, C., Aubier, M.G., Daigne, G., Genova, F., Leblanc, Y., Lecacheux, A., de la Noe, J., Moller-Pedersen, B.: A new high-grain, broadband, steerable array to study Jovian decametric emission. Icarus 43, 399-407 (1980)

7. Bottke, W.F., Morbidelli, A., Jedicke, R., Petit, J.M., Levison, H.F., Michel, P., Metcalfe, T.S.: Debiased orbital and absolute magnitude distribution of the near-Earth objects. Icarus 156, 399-433 (2002)

8. Briand, C., Zaslavsky, A., Maksimovic, M., Zarka, P., Lecacheux, A., Rucker, H.O., Konovalenko, A.A., Abranin, E.P., Dorovsky, V.V., Stanislavsky, A.A., Melnik, V.N.: Faint solar radio structures from decametric observations. Astron. Astrophys. 490, 339-344 (2008)

9. Brown, P., Spalding, R.E., ReVelle, D.O., Tagliaferri, E., Worden, S.P.: The flux of small nearEarth objects colliding with the Earth. Nature 420, 294-296 (2002)

10. Bruck, Yu.M., Ustimenko, B.Yu.: Decametric radio emission from four pulsars. Nature 260, 766-767 (1976)

11. Campbell-Brown, M.D.: High resolution radiant distribution and orbits of sporadic radar meteoroids. Icarus 196, 144-163 (2008)

12. Cecconi, B.: Goniopolarimetric techniques for low-frequency radio astronomy in space. In: Huber, M.C.E., Pauluhn, A., Culhane, J.L., Timothy, J.G., Wilhelm, K., Zehnder, A. (eds.) Observing Photons in Space. ISSI Scientific Reports Series, pp. 263-277. Springer (2010)

13. Committee on the planetary science decadal survey: Vision and Voyages for Planetary Science in the Decade 2013-2022. National Research Council, National Academies Press, Washington, DC (2010)

14. Elphic, R.C., Chu, P., Hahn, S., James, M.R., Lawrence, D.J., Prettyman, T.H., Johnson, J.B., Podgorney, R.K.: Surface and downhole prospecting tools for planetary exploration: tests of neutron and gamma ray probes. Astrobiology 8, 639-652 (2008)

15. Dulk, G.A., Erickson, W.C., Manning, R., Bougeret, J.-L.: Calibration of low-frequency radio telescopes using the galactic background radiation. Astron. Astrophys. 365, 294-300 (2001)

16. Dyal, P., Parkin, C.W., Daily, W.D.: Magnetism and the interior of the Moon. Rev. Geophys. 12, 568-591 (1974)

17. Garcia, R.F., Gagnepain-Beyneix, J., Chevrot, S., Lognonné, P.: Very preliminary reference Moon model. Phys. Earth Planet. Int. doi:10.1016/j.pepi.2011.06.015 (2011)

18. Garrick-Bethell, I, Zuber, M.T.: Elliptical structure of the lunar South Pole-Aitken basin. Icarus 204, 399-408 (2009)

19. Gough, D.I., Ingham, M.R.: Interpretation methods for magnetometer arrays. Rev. Geophys. 21, 805-827 (1983)

20. Grimm, R.E., Delory, G.T.: Next-generation electromagnetic sounding of the Moon. Adv. Space Res. (2011, under review)

21. Grott, M., Knollenberg, J., Krause, C.: Apollo lunar heat flow experiment revisited: a critical reassessment of the in situ thermal conductivity determination. J. Geophys. Res. 115, E11005 (2010) 
22. Hill, K., Parker J., Born, G.H., Demandante, N.: A lunar L2 navigation, communication, and gravity mission. In: AIAA/AAS Astrodynamics Specialist Conference and Exhibit, 2124 August 2006, AIAA 2006-6662, Keystone, Colorado

23. Hood, L.L.: Geophysical constraints on the lunar interior. In: Hartmann, W.K., Phillips, R.J., Taylor, G.J. (eds.) Origin of the Moon, pp. 361-410. Lunar and Planetary Institute, Houston (1986)

24. Hood, L.L., Herbert, F., Sonett, C.P.: The deep lunar electrical conductivity profile: structural and thermal inferences. J. Geophys. Res. 87, 5311-5326 (1982)

25. Hood, L.L., Sonett, C.P.: Limits on the lunar temperature profile. Geophys. Res. Lett. 9, 37-40 (1982)

26. Ivanov, B.A.: Earth/Moon impact rate comparison: searching constraints for lunar secondary/primary cratering proportion. Icarus 183, 504-507 (2006)

27. Jester, S., Falcke, S.: Science with a lunar low-frequency array: from the dark ages of the Universe to nearby exoplanets. New Astron. Rev. 53, 1-26 (2009)

28. Jolliff, B.L., Gillis, J.J., Haskin, L.A., Korotev, R.L., Wieczorek, M.W.: Major lunar crustal terranes: surface expressions and crust-mantle origins. J. Geophys. Res. 105, 4197-4216 (2000)

29. Jolliff, B., Wieczorek, M., Shearer, C., Neal, C. (eds.): New views of the Moon. Rev. Mineral. Geochem. 60, 721 (2006)

30. Kaiser, M.L.: A low-frequency radio survey of the planets with RAE 2. J. Geophys. Res. 82, 1256-1260 (1977)

31. Khan, A., Connolly, J.A.D., Olsen, N., Mosegaard, K.: Constraining the composition and thermal state of the Moon from an inversion of electromagnetic lunar day-side transfer functions. Earth Planet. Sci. Lett. 248, 579-598 (2006)

32. Konovalenko, A.: Ukraine decameter wave radio astronomy systems and their perspectives. In: Stone, R.G., Weiler, K.W., Goldstein, M.L., Bougerot. J.-L. (eds.) Radio Astronomy at Long Wavelengths, Geophysical monograph, vol. 119, pp. 311-320. American Geophysical Union, Washington, DC (2000)

33. Langseth, M.G., Keihm, S.J. Peters, K.: Revised lunar heat-flow values. In: Proc. Lunar Sci. Conf. 7th, 3143-3171 (1976)

34. Lawrence, D.J., Elphic, R.C., Feldman, W.C., Prettyman, T.H., Gasnault, O., Maurice, S.: Small-area thorium features on the lunar surface. J. Geophys. Res. 108, 5102 (2003)

35. Le Feuvre, M., Wieczorek, M.A.: Nonuniform cratering of the Moon and a revised crater chronology of the inner solar system. Icarus 214, 1-20 (2011)

36. Le Feuvre, M., Wieczorek, M.A.: Nonuniform cratering of the terrestrial planets. Icarus 197, 291-306 (2008)

37. Lecacheux, A., Konovalenko, A.A., Rucker, H.O.: Using large radio telescopes at decametre wavelengths. Planet. Space Sci. 52, 1357-1374 (2004)

38. Lognonné, P., Giardini, D., Banerdt, B., Gagnepain-Beyneix, J., Mocquet, A., Spohn, T., Karczewski, J.F., Schibler, P., Cacho, S., Pike, W.T., Cavoit, C., Desautez, A., Favede, M., Gabsi, T., Simoulin, L., Striebig, N., Campillo, M., Deschamp, A., Hinderer, J., Leveque, J.J., Montagner, J.P., Rivera, L., Benz, W., Breuer, D., Defraigne, P., Dehant, V., Fujimura, A., Mizutani, H., Oberst, J.: The NetLander very broad band seismometer. Planet. Space Sci. 48, 1289-1302 (2000)

39. Lognonné, P., Le Feuvre, M., Johnson, C.L., Weber, R.C.: Moon meteoritic seismic hum: steady state prediction. J. Geophys. Res. 114, E12003 (2009)

40. Lognonné P., Johnson, C.L.: Planetary seismology. Treatise on Geophysics 10, 69-122 (2007)

41. Lucey, P., Korotev, R.L., Gillis, J.J., Taylor, L.A., Lawrence, D., Campbell, B.A., Elphic, R., Feldman, B., Hood, L.L., Hunten, D., Mendillo, M., Noble, S., Papike, J.J., Reedy, R.C., Lawson, S., Prettyman, T., Gasnault, O., Maurice, S.: Understanding the lunar surface and space-Moon interactions. Rev. Mineral. Geochem. 60, 83-219 (2006)

42. Mann, G., Jansen, F., MacDowall, R.J., Kaiser, M.L., Stone, R.G.: A heliospheric density model and type III radio bursts. Astron. Astrophys. 348, 614-620 (1999)

43. Mel'nik, V.N., Konovalenko, A.A., Rucker, H.O., Rutkevych, B.P., Dorovskyy, V.V., Abranin, E.P., Brazhenko, A.I., Stanislavskyy, A.A., Lecacheux, A.: Decameter type III like bursts. Solar Phys. 250, 133-145 (2008) 
44. Mimoun, D., Lognonné, P., Giardini, D., Christensen, U., Zweifel, P., Mance, D., Roll, R., Bierwirth, M., de Raucourt, S., Gagnepain-Beyneix, J., Tillier, S., Nebut, T., Robert, O., Pot, O., Schibler, P., SEIS Team: MOON SEIS: design and performances of a very broadband seismometer for future lunar missions. Ground-Based Geophysics on the Moon, LPI Contribution No. 1530, 3017. Tempe, Arizona, 21-22 January (2010)

45. Morgan, T.: International lunar network working group 1: International Lunar Network core instruments working group. http://iln.arc.nasa.gov/ (2009)

46. Nakamura, Y., Latham, G.V., Dorman, H.J.: Apollo lunar seismic experiment-a final summary. Proc. Lunar Planet. Sci. Conf. 13; J. Geophys. Res. 87, A117-A123 (1982)

47. Nemtchinov, I.V., Shuvalov, V.V. Artem'eva, N.A., Ivanov, B.A., Kosarev, I.B., Trubetskaya, I.A.: Light flashes caused by meteoroid impacts on the lunar surface. Solar System Res. 32, 99-114 (1998)

48. Oberst, J., Nakamura, Y.: A search for clustering among the meteoroid impacts detected by the Apollo lunar seismic network. Icarus 91, 315-325 (1991)

49. Oberst, J., Nakamura, Y.: Monte Carlo simulations of the diurnal variation in seismic detection rate of sporadic meteoroid impacts on the Moon. Proc. Lunar Planet. Sci. Conf. 19, 615-625 (1989)

50. Oberst, J., Flohrer, J., Elgner, S., Maue, T., Margonis, A., Schroedter, R., Tost, W., Buhl, M., Ehrich, J., Christou, K., Koschny, D.: The Smart Panoramic Optical Sensor Head (SPOSH) a camera for observations of transient luminous events on planetary night sides. Planet. Space Sci. 59, 1-9 (2011)

51. Ortiz, J.L., Aceituno, F.J., Quesada, J.A., Aceituno, J., Fernandez, M., Santos-Sanz, P., TrigoRodriguez, J.M., Llorca, J., Martin-Torres, F.J., Montanes-Rodriguez, P., Palle, E.: Detection of sporadic impact flashes on the Moon: implications for the luminous efficiency of hypervelocity impacts and derived terrestrial impact rates. Icarus 184, 319-326 (2006)

52. Ortiz, J.L., Sada, P.V., Bellot Rubio, L.R., Aceituno, F.J., Aceituno, J., Gutiérrez, P.J., Thiele, U.: Optical detection of meteoroidal impacts on the Moon. Nature 405, 921-923 (2000)

53. Parker, J.: Families of low-energy lunar halo transfers. In: AAS/AIAA SpaceFlight Mechanics Conference, AAS06-132, Tampa, Florida, 22-26 January (2006)

54. Parker, J.: Low-energy ballistic lunar transfers. Ph.D. Thesis, University of Colorado, Department of Aerospace Engineering Sciences (2007)

55. Pieters, C.M., Besse, S., Boardman, J., Buratti, B., Cheek, L., Clark, R.N., Combe, J.P., Dhingra, D., Goswami, J.N., Green, R.O., Head, J.W., Isaacson, P., Klima, R., Kramer, G., Lundeen, S., Malaret, E., McCord, T., Mustard, J., Nettles, J., Petro, N., Runyon, C., Staid, M., Sunshine, J., Taylor, L.A., Thaisen, K., Tompkins, S., Whitten, J.: Mg-spinel lithology: a new rock type on the lunar farside. J. Geophys. Res. 116, E00G08 (2011)

56. Popov, M.V., Kuz'min, A., Ul'yanov, O., Deshpande, A., Ershov, A., Zakharenko, V., Kondrat'ev, V.I., Kostyuk, S.V., Losovskii, B., Soglasnov, V.: Instant radio spectra of giant pulses from the Crab pulsar from decimeter to decameter wavelengths. Astron. Reports 50, 562-568 (2006)

57. Pradier, A., Fisackerly, R., Houdou, B., Phillipe, C., Carpenter, J., de Rosa, D., Espinasse, S., Gardini, B., Henn, N.: The first European lunar lander and the ESA-DRL approach to its development. IAC 61, IAC-10.A3.2B.8 (2010)

58. Prettyman, T.H., Hagerty, J.J., Elphic, R.C., Feldman, W.C., Lawrence, D.J., McKinney, G.W., Vaniman, D.T.: Elemental composition of the lunar surface: analysis of gamma ray spectroscopy data from Lunar Prospector. J. Geophys. Res. 111, E12007 (2006)

59. Pritchard, J.R., Loeb, A.: Constraining the unexplored period between the dark ages and reionization with observations of the global $21 \mathrm{~cm}$ signal. Phys. Rev. D 82, 023006 (2010)

60. Shearer, C., Tahu, G.: Lunar Geophysical Network (LGN). Planetary science decadal survey, mission concept study report, http://sites.nationalacademies.org/SSB/SSB_059331 (2010)

61. Simpson, F., Bahr, K.: Practical Magnetotellurics. Cambridge Univ. Press, 270 pp. (2005)

62. Smith, D.E., Zuber, M.T., Neumann, G.A. Lemoine, F.G., Mazarico, E., Torrence, M.H., McGarry, J.F., Rowlands, D.D., Head, J.W., Duxbury, T.H., Aharonson, O., Lucey, P.G., Robinson, M.S., Barnouin, O.S., Cavanaugh, J.F., Sun, X., Liiva, P., Mao, D.-d., Smith, J.C., Bartels, A.E.: Initial observations from the Lunar Orbiter Laser Altimeter (LOLA). Geophys. Res. Lett. 37, L18204 (2010)

63. Sonett, C.P.: Electromagnetic induction in the Moon. Rev. Geophys. 20, 411-455 (1982) 
64. Spohn, T., Konrad, W., Breuer, D., Ziethe, R.: The longevity of lunar volcanism: implications of thermal evolution calculations with $2 \mathrm{D}$ and $3 \mathrm{D}$ mantle convection models. Icarus 149, 54-65 (2001)

65. Spohn, T., Grott, M., Richter, L., Knollenberg, J., Smrekar, S.E.: HP3 Instrument Team: Measuring heat flow on the Moon-the heat flow and physical properties package HP3. Ground-based Geophysics on the Moon, LPI Contribution No. 1530, 3016. Tempe, Arizona, 21-22 Jan (2010)

66. Suggs, R.M., Cooke, W.J., Suggs, R.J., Swift, W.R., Hollon, N.: The NASA lunar impact monitoring program. Earth Moon Planets 102, 293-298 (2008)

67. Takahashi, Y.D.: A concept for a simple radio observatory at the lunar south pole. Adv. Space Res. 31, 2473-2478 (2003)

68. Vyshlov, A.S., Savich, N.A., Vasilyev, M.B., Samoznaev, L.N., Sidorenko, A.I., Shtern, D.Y.: Some results of cislunar plasma research. NASA Spec. Publ. 397, 81-85 (1976)

69. Warren, P.: "New" lunar meteorites: implications for composition of the global lunar surface, lunar crust, and the bulk Moon. Meteor. Planet. Sci. 40, 477-506 (2005)

70. Weber, R.C., Lin, P.-Y., Garnero, E.J., Williams, Q., Lognonné, P.: Seismic detection of the lunar core. Science 331, 309-312 (2011)

71. Wieczorek, M.: International lunar network working group 3: international lunar network. Site selection working group final report (2011, in preparation)

72. Wieczorek, M.A., Phillips, R.J.: The "Procellarum KREEP Terrane": implications for mare volcanism and lunar evolution. J. Geophys. Res. 105, 20417-20430 (2000)

73. Wieczorek, M.A., Jolliff, B.L., Khan, A., Pritchard, M.E., Weiss, B.P., Williams, J.G., Hood, L.L., Righter, K., Neal, C.R., Shearer, C.K., McCallum, I.S., Tompkins, S., Hawke, B.R., Peterson, C., Gillis, J.J., Bussey, B.: The constitution and structure of the lunar interior. Rev. Mineral. Geochem. 60, 221-364 (2006)

74. Yamada, R., Yamada, I., Shiraishi, H., Tanaka, S., Takagi, Y., Kobayashi, N., Takeuchi, N., Ishihara, Y., Murakami, H., Yomogida, K., Koyama, J., Fujimura, A., Mizutani, H.: Capability of the penetrator seismometer system for lunar seismic event observation. Planet. Space Sci. 57, 751-763 (2009)

75. Yamada, R., Garcia, R.F., Lognonné, P., Le Feuvre, M., Calvet, M., Gagnepain-Beyneix, J.: Optimisation of seismic network design: application to a geophysical international lunar network. Planet. Space Sci. 59, 343-354 (2011)

76. Yanagisawa, M., Kisaichi, N.: Lightcurves of 1999 Leonid impact flashes on the Moon. Icarus 159, 31-38 (2002)

77. Zarka, P., Lamy L., Cecconi, B., Prangé, R., Rucker, H.O.: Modulation of Saturn's radio clock by solar wind speed. Nature 450, 265-267 (2007)

78. Zarka, P.: Auroral radio emissions at the outer planets: observations and theories. J. Geophys. Res. 103, 20159-20194 (1998)

79. Zarka, P.: Plasma interactions of exoplanets with their parent star and associated radio emissions. Planet. Space Sci. 55, 598-617 (2008)

80. Zarka, P., Tagger, M.: Planetary science and exoplanet detection at low radio frequencies. Towards a European infrastructure for lunar observatories II. Bremen, Germany, 23-24 November (2006) 